ULB-TH-04/11, IFUP-TH/2004-5

\title{
NONABELIAN MONOPOLES
}

\author{
Roberto AUZZI ${ }^{(1,3)}$, Stefano BOLOGNESI ${ }^{(1,3)}$, \\ Jarah EVSLIN ${ }^{(4)}$, Kenichi KONISHI ${ }^{(2,3)}$, Hitoshi MURAYAMA ${ }^{(5) *}$

\begin{abstract}
:
We study topological as well as dynamical properties of BPS nonabelian magnetic monopoles of Goddard-Nuyts-Olive-Weinberg type in $G=S U(N), U S p(2 N)$ and $S O(N)$ gauge theories, spontaneously broken to nonabelian subgroups $H$. We find that monopoles transform under the group dual to $H$ in a tensor representation of rank determined by the corresponding element in $\pi_{1}(H)$. When the system is embedded in a $\mathcal{N}=2$ supersymmetric theory with an appropriate set of flavors with appropriate bare masses, the BPS monopoles constructed semiclassically persist in the full quantum theory. This result supports the identification of "dual quarks" found at $r$-vacua of $\mathcal{N}=2$ theories with the nonabelian magnetic monopoles. We present several consistency checks of our monopole spectra.
\end{abstract}

June 22, 2017

*On leave of absence from Department of Physics, University of California, Berkeley, CA 94720, $U S A$ 


\section{Introduction}

After many years of investigations [1]-5] the dynamical roles that nonabelian monopoles can play in physically interesting gauge theories are finally being understood. Although their presence in classical examples of conformally invariant $\mathcal{N}=4$ theories and in more recent $\mathcal{N}=1$ supersymmetric models with Seiberg duals [12] is by now well known, monopoles with nonabelian gauge symmetry manifest themselves most clearly (e.g., as the infrared degrees of freedom) in softly broken $\mathcal{N}=2$ gauge theories coupled to fundamental matter hypermultiplets [13]-15].

In particular, a series of papers on this class of theories with gauge groups $S U(N)$, $U S p(2 N)$ and $S O(N)$ and various numbers of flavors of matter multiplets [16-18] systematically investigated the infrared fate of these monopoles in every vacuum. The "dual quarks" appearing as the low-energy degrees of freedom in certain vacua of these theories, which are charged under the unbroken nonabelian $S U(r) \subset G$, can be identified [19] with the semiclassical monopoles studied earlier by Goddard, Nuyts and Olive [4] and also by E. Weinberg [6]. All of the confining vacua of strongly coupled $U S p(2 N)$ and $S O(N)$ theories with flavors and without bare quark masses involve these objects in a deformed SCFT [17, 18, 20].

Recently, with A. Yung, we have explored the properties of nonabelian BPS vortices appearing in the same class of models [21]. In particular, continuous transformations among the degenerate vortex solutions (vortex zero modes) were explicitly constructed, showing the true nonabelian nature of these solitons. The analysis is done in a region of large bare quark masses where the semi-classical approximation holds, and yet the whole result is quantum mechanically valid when the model is embedded in the $\mathcal{N}=2$ theory [21]. ${ }^{1}$

In the present paper, our attention will be focused on the topological and dynamical properties of nonabelian monopoles themselves, in a wide class of $\mathcal{N}=2$ gauge theories with $S U(N), U S p(2 N)$ and $S O(N)$ gauge groups, spontaneously broken to various nonabelian subgroups $H$. In particular for each symmetry breaking pattern the minimal monopoles are identified and their nonabelian and abelian charges

\footnotetext{
${ }^{1}$ There are papers related to ours by Hanany and Tong [22, and also by Kneipp 23]. Interesting recent articles [24, 25] furthermore relate the vortex dynamics to that of 4D gauge theory itself.
} 
determined. The monopole-vortex flux matching argument given in [26] is crucial in demonstrating, albeit indirectly, that a continuous set of these monopoles exist, forming a multiplet of the dual gauge group, $\tilde{H}$.

This paper is organized as follows. We start our analysis (Section 2) by reviewing the properties of BPS "nonabelian" monopole solutions in a bosonic theory with a spontaneously broken $S U(N+1) \rightarrow S U(N) \times U(1)$ symmetry. We then show that when the model is embedded in a $\mathcal{N}=2$ supersymmetric theory [13]-[15], the semiclassical solutions of the bosonic model acquire a quantum mechanical meaning. The quantum mechanical aspects of nonabelian monopoles and in particular the importance of the massless flavors in the underlying theory, are discussed more thoroughly and in a more general context in Section 3. In Section 4 we establish their topological stability and determine their abelian magnetic charges with respect to the minimal Dirac quantum, based on homotopy group analysis, for $G=S U(N), U S p(2 N)$ or $S O(N)$ and for various choices of $H$. In Section 5 we present an explicit construction of these minimal monopoles. We summarize our results in Section 6, presenting also some crosschecks of consistency of our results. In Appendix A the general formulae due to E. Weinberg and to Goddard-Nuyts-Olive are reviewed and somewhat streamlined, which helps as a reference for other parts of the paper. For completeness and for convenience we review also the root vector systems and Cartan subalgebras of $S U, S O$ and $U S p$ Lie algebras in Appendix B.

\section{Nonabelian BPS Monopoles in $S U(N+1)$ Theories}

For illustration we begin our discussion by briefly reviewing the properties of the monopoles arising in a system with symmetry breaking $S U(N+1) \rightarrow S U(N) \times U(1)$.

\subsection{Bosonic $S U(N+1)$ theory with an adjoint scalar}

We are interested in the standard $S U(N+1)$ model

$$
\mathcal{L}=\frac{1}{4 g^{2}}\left(F_{\mu \nu}^{A}\right)^{2}+\frac{1}{g^{2}}\left|\left(\mathcal{D}_{\mu} \phi\right)^{A}\right|^{2}-V(\phi)
$$


with a complex adjoint scalar field $\phi$. Let us assume that the potential is minimized by an adjoint scalar VEV of the form

$$
\langle\phi\rangle=\left(\begin{array}{cccc}
v & 0 & \ldots & 0 \\
0 & v & \ldots & 0 \\
\vdots & \vdots & \ddots & \vdots \\
0 & 0 & \ldots & -N v
\end{array}\right)=\left(\begin{array}{cc}
v \cdot \mathbf{1}_{N \times N} & \\
& -N v
\end{array}\right)
$$

Such a VEV breaks the gauge symmetry as

$$
S U(N+1) \rightarrow \frac{S U(N) \times U(1)}{\mathbb{Z}_{N}}=U(N),
$$

where the $\mathbb{Z}_{N}$ factor arises because the $n$th roots of unity in $U(1)$ also lie in $S U(N)$.

The stability of a monopole is guaranteed by the topological nontriviality of both its $S U(N+1)$-valued adjoint scalar Higgs field and its gauge fields. The Higgs VEV, evaluated on a 2 -sphere surrounding any monopole, provides a map from the 2 -sphere to the space of orbits of the unbroken gauge group $U(N)$ in the original $S U(N+1)$. The Higgs VEV is therefore a representative of $\pi_{2}(S U(N+1) / U(N))$.

On the other hand, The gauge field configuration describes a $U(N)$ gauge bundle over the spacetime with the monopole deleted, which can be contracted to a 2 -sphere. Such a gauge bundle may be trivialized over the northern and southern hemispheres, and so is entirely characterized by the transition function on the equator. The transition function is a map from the equatorial circle to the gauge group $U(N)$, and so it is a representative of $\pi_{1}(U(N))$.

The long exact sequence for homotopy groups of fibrations assures that these two classifications of monopoles agree. The two homotopy groups are both the group of integers

$$
\pi_{2}\left(\frac{S U(N+1)}{U(N)}\right)=\pi_{1}(U(N))=\mathbb{Z}
$$

and so these monopoles are topologically stable and may carry any integral charge. We will see later that if the original gauge symmetry $G$ is not simply connected then the long exact sequence yields more topologically distinct gauge field configurations than Higgs configurations. These extra gauge field configurations are singular Diraclike monopoles that exist in the $G$ gauge theory even without symmetry breaking, and are not the nonsingular monopoles of interest in this paper. For semi-simple $G$ the fundamental group is torsion and so the extra monopole charges are pure torsion and the extra monopoles are not BPS. 
The mass of a BPS monopole may be read from the Hamiltonian

$$
H=\int d^{3} x\left[\frac{1}{4 g^{2}}\left(F_{i j}^{A}\right)^{2}+\frac{1}{g^{2}}\left|\mathcal{D}_{i} \phi^{A}\right|^{2}\right]=\int d^{3} x\left[\frac{1}{4 g^{2}}\left(F_{i j}^{A}\right)^{2}+\frac{1}{2 g^{2}}\left|\mathcal{D}_{i} \phi^{A}\right|^{2}\right]
$$

where in the final expression we have kept only the real part of $\phi^{A}$. Rewriting the Hamiltonian as

$$
H=\int d^{3} x\left[\frac{1}{4 g^{2}}\left|F_{i j}^{A} \pm \epsilon_{i j k}\left(\mathcal{D}_{k} \phi\right)^{A}\right|^{2} \pm \frac{1}{2} \partial_{k}\left(\epsilon_{i j k} F_{i j}^{A} \phi^{A}\right)\right]
$$

BPS monopole 28] configurations are seen to satisfy the nonabelian Bogomolny equations:

$$
B_{k}^{A}=-\left(\mathcal{D}_{k} \phi\right)^{A} ; \quad B_{k}^{A}=\frac{1}{2} \epsilon_{i j k} F_{i j}^{A} .
$$

The BPS bound on the monopole mass is given by the following integral, performed on the 2-sphere at $r=\infty$ :

$$
H=\int d S \cdot\left(\phi^{A} \mathbf{B}^{A}\right)=\frac{2 \pi}{g} 3 v k, \quad k=1,2, \ldots
$$

More explicitly, a monopole solution can be found by choosing an $S U(2)$ subgroup:

$$
S_{1}=\frac{1}{2}\left(\begin{array}{cccc}
0 & 0 & \ldots & 1 \\
0 & 0 & \ldots & 0 \\
\vdots & \vdots & \ddots & \vdots \\
1 & 0 & \ldots & 0
\end{array}\right) ; \quad S_{2}=\frac{1}{2}\left(\begin{array}{cccc}
0 & 0 & \ldots & -i \\
0 & 0 & \ldots & 0 \\
\vdots & \vdots & \ddots & \vdots \\
i & 0 & \ldots & 0
\end{array}\right) ; \quad S_{3}=\frac{1}{2}\left(\begin{array}{cccc}
1 & 0 & \ldots & 0 \\
0 & 0 & \ldots & 0 \\
\vdots & \vdots & \ddots & \vdots \\
0 & 0 & \ldots & -1
\end{array}\right)
$$

The monopole solution is then given by [, [19]:

$$
\begin{gathered}
\phi=\left(\begin{array}{ccccc}
-\frac{N-1}{2} v & 0 & \ldots & 0 & 0 \\
0 & v & 0 & \ldots & 0 \\
0 & 0 & v & \ldots & 0 \\
\vdots & \vdots & \vdots & \ddots & \vdots \\
0 & 0 & 0 & \ldots & -\frac{N-1}{2} v
\end{array}\right)+(N+1) v(\vec{S} \cdot \widehat{r}) \phi(r), \\
\vec{A}(r)=\vec{S} \wedge \widehat{r} \frac{A(r)}{g}
\end{gathered}
$$

where $\phi(r)$ and $A(r)$ are 't Hooft-BPS functions with $\phi(\infty)=1, \phi(0)=0, A(\infty)=$ $-1 / r$. To compute the mass, using Eq. (2.8) one needs the following property:

$$
\frac{1}{2} \epsilon^{i j k} F_{i j} \widehat{r}_{k}=-\frac{\vec{S} \cdot \widehat{r}}{g r^{2}}
$$


yielding the result

$$
M=\frac{2 \pi(N+1) v}{g} .
$$

In order to calculate the abelian charge with respect to the $U(1)$ factor in Eq.(2.3) we need to first calculate the total magnetic flux sourced by the minimal monopole. Normalizing the flux by the norm of $\phi$, one finds

$$
F_{m}=\int_{S^{2}} d \mathbf{S} \cdot \frac{\operatorname{Tr} \phi \mathbf{B}}{\frac{1}{\sqrt{2}}\left(\operatorname{Tr} \phi^{2}\right)^{1 / 2}}=\frac{2 \pi(N+1)}{g \sqrt{N(N+1)} / \sqrt{2}}=\frac{2 \pi}{g} \sqrt{\frac{2(N+1)}{N}} .
$$

This should be equal to $4 \pi g_{m}$, so

$$
g_{m}=\frac{1}{g} \sqrt{\frac{N+1}{2 N}} .
$$

On the other hand, the electric coupling of the $A_{\mu}^{0}$ field to the matter in the fundamental representation of $S U(N+1)$ is through the minimum coupling constant

$$
e_{0}=\frac{g}{\sqrt{2 N(N+1)}}
$$

as

$$
t^{0}=\frac{1}{\sqrt{2 N(N+1)}}\left(\begin{array}{ll}
\mathbf{1}_{N \times N} & \\
& -N
\end{array}\right) .
$$

Thus the minimum magnetic charge, in terms of the unit electric charge, is

$$
g_{m}=\frac{1}{2 N e_{0}}
$$

which is $1 / N$ of the charge of Dirac's $U(1)$ monopole [29]. In Sec [ we will see that this factor of $N$ is the degree of the embedding of the fundamental group of the unbroken $U(1)$ into that of the unbroken gauge group.

Clearly, the choice made above (2.9) is nothing but one of the $N$ possibilities. By using the $S U(2)$ subgroups lying in the $(i, N+1)-2 \times 2$ subspaces, $i=1,2, \ldots, N$, we finds $N$ degenerate monopoles with identical masses and charges. This is the right multiplicity for these monopoles to belong to the fundamental representation of the dual $S U(N)$ magnetic group.

\subsection{Embedding the System in $\mathcal{N}=2$}

The fact that the monopoles associated with the symmetry breaking $G \stackrel{\langle\phi\rangle \neq 0}{\longrightarrow} H$ semiclassically form a degenerate multiplet, however, does not in itself prove that they are 
nonabelian monopoles. The main problem is that the "unbroken" gauge group $H$ (in the case just considered, $U(N)$ ) can dynamically break down to an abelian subgroup. Whether such a dynamical breaking occurs depends on the details of the quantum system and it is in general difficult to decide what actually takes place.

In $\mathcal{N}=2$ supersymmetric models a definite answer can be given. If the model above is embedded in the pure $\mathcal{N}=2$ theory

$$
\mathcal{L}=\frac{1}{8 \pi} \operatorname{Im} \tau_{c l}\left[\int d^{4} \theta \Phi^{\dagger} e^{V} \Phi+\int d^{2} \theta \frac{1}{2} W W\right],
$$

$\left(\tau_{c l} \equiv \frac{\theta_{0}}{\pi}+\frac{8 \pi i}{g_{0}^{2}}\right)$, the $S U(N)$ sector left "unbroken" by the adjoint scalar VEV (2.2) describes a pure $\mathcal{N}=2 S U(N)$ theory, which becomes strongly coupled at low energies and is dynamically broken to the maximal Abelian subgroup $U(1)^{N-1}[14,15,27$.

In order to preserve an unbroken subgroup $H$ we couple the theory to $N_{f}$ hypermultiplets (quarks). The Lagrangian of this theory has the structure

$$
\begin{gathered}
\mathcal{L}=\frac{1}{8 \pi} \operatorname{Im} S_{c l}\left[\int d^{4} \theta \Phi^{\dagger} e^{V} \Phi+\int d^{2} \theta \frac{1}{2} W W\right]+\mathcal{L}^{(\text {quarks })}+\int d^{2} \theta \mu \operatorname{Tr} \Phi^{2} \\
\mathcal{L}^{(\text {quarks })}=\sum_{i}\left[\int d^{4} \theta\left\{Q_{i}^{\dagger} e^{V} Q_{i}+\tilde{Q}_{i} e^{-V} \tilde{Q}_{i}^{\dagger}\right\}+\int d^{2} \theta\left\{\sqrt{2} \tilde{Q}_{i} \Phi Q^{i}+m \tilde{Q}_{i} Q^{i}\right\}\right.
\end{gathered}
$$

where $m$ is the (common) bare mass of the quarks and we have defined the complex coupling constant

$$
S_{c l} \equiv \frac{\theta_{0}}{\pi}+\frac{8 \pi i}{g_{0}^{2}}
$$

The parameter $\mu$ is the mass of the adjoint chiral multiplet, which breaks the supersymmetry to $\mathcal{N}=1$.

In order to discuss unconfined monopoles, however, one must set $\mu=0$ and so preserve the full $\mathcal{N}=2$ supersymmetry. After eliminating the auxiliary fields the bosonic Lagrangian becomes

$$
\mathcal{L}=\frac{1}{4 g^{2}} F_{\mu \nu}^{2}+\frac{1}{g^{2}}\left|\mathcal{D}_{\mu} \phi\right|^{2}+\left|\mathcal{D}_{\mu} Q\right|^{2}+\left|\mathcal{D}_{\mu} \overline{\tilde{Q}}\right|^{2}+\mathcal{L}_{1}+\mathcal{L}_{2}
$$

where

$$
\begin{aligned}
\mathcal{L}_{1} & =-\frac{1}{8} \sum_{A}\left[\frac{1}{g^{2}}(-i) f_{A B C} \phi_{B}^{\dagger} \phi_{C}+Q^{\dagger} t^{A} Q-\tilde{Q} t^{A} \tilde{Q}^{\dagger}\right]^{2} \\
& =-\frac{1}{8} \sum_{A}\left(t_{i j}^{A}\left[\frac{1}{g^{2}}(-2)\left[\phi^{\dagger}, \phi\right]_{j i}+Q_{j}^{\dagger} Q_{i}-\tilde{Q}_{j} \tilde{Q}_{i}^{\dagger}\right]\right)^{2} ;
\end{aligned}
$$




$$
\begin{aligned}
\mathcal{L}_{2} & =-g^{2}\left|\sqrt{2} \tilde{Q} t^{A} Q\right|^{2}-\tilde{Q}[m+\sqrt{2} \phi][m+\sqrt{2} \phi]^{\dagger} \tilde{Q}^{\dagger} \\
& -Q^{\dagger}[m+\sqrt{2} \phi]^{\dagger}[m+\sqrt{2} \phi] Q .
\end{aligned}
$$

In the construction of the monopole solutions we shall consider only the VEVs and fluctuations around them which satisfy

$$
\left[\phi^{\dagger}, \phi\right]=0, \quad Q_{i}=\tilde{Q}_{i}^{\dagger},
$$

and hence $\mathcal{L}_{1}$ can be set to zero.

We are interested in the vacua which would survive the above $(\mu \neq 0)$ perturbation to $\mathcal{N}=1$. These vacua are parametrized by the integer $r$, which is the rank of the unbroken nonabelian gauge symmetry plus one [16, 17]. In order to exhibit the symmetry breaking $S U(N+1) \rightarrow S U(N) \times U(1)$ one may choose the adjoint VEV to be

$$
\langle\phi\rangle=-\frac{m}{\sqrt{2}} \operatorname{diag}(1,1, \ldots, 1,-N),
$$

namely, as in Eq.(2.2) with $v=-\frac{m}{\sqrt{2}}$. Together with the vanishing squark VEVs

$$
\left\langle Q_{i}\right\rangle=\left\langle\tilde{Q}_{i}\right\rangle=0
$$

this is easily seen to give vanishing contribution to the bosonic Lagrangian (2.23). (2.27) and (2.28) represent a supersymmetric vacuum as $\mathcal{L}_{1}=\mathcal{L}_{2}=0$.

One is left with the task of minimizing the first two terms of the bosonic Lagrangtian (2.23): the rest of the discussion is exactly as in Sec. 2.1] semiclassically nothing changes. Quantum mechanically, however, there is an important difference. In the vacuum characterized by the VEV (2.27) there are $N_{f}$ massless quarks (and squarks) in the fundamental representation of $S U(N)$. This ensures that the subgroup $S U(N)$ is non-asymptotically free and remains unbroken in the infrared if $2(N+1)>N_{f} \geq 2 N$. This strong restriction on the number of flavors may be significantly relaxed in cases in which the unbroken group is smaller. In this way one finds that the only real restriction is that the number of flavors be at least equal to $2 r$ if the monopole transforms in the fundamental representation of $S U(r)$. (See e.g., Eq.(3.2) $)$.)

\section{Quantum Nonabelian Monopoles}

The above example of the $S U(N+1)$ model nicely illustrates the fact that a semiclassical treatment alone is not enough to ensure that the set of apparently degenerate 
monopoles associated with the symmetry breaking $G \stackrel{\langle\phi\rangle \neq 0}{\longrightarrow} H$ are truly nonabelian. The reason is that the "unbroken" gauge group $H$ may well dynamically break down to an abelian subgroup. If this occurs, one has only an approximately degenerate set of monopoles whose masses differ by e.g., $O\left(\frac{\Lambda^{2}}{\langle\phi\rangle}\right)$. For this reason, the very concept of nonabelian monopoles is never really semi-classical, in sharp contrast to the case of abelian monopoles. Only if the "unbroken" gauge group $H$ is not further broken dynamically do the unconfined (topologically stable) nonabelian monopoles and dual gauge bosons appear in the quantum theory.

Another subtlety is that it is not justified to study the system $G \stackrel{\langle\phi\rangle \neq 0}{\longrightarrow} H$ with a nonabelian subgroup $H$ as a limiting situation of a maximal breaking, $G \stackrel{\langle\phi\rangle \neq 0}{\longrightarrow} U(1)^{R}$, where $R$ is the rank of the group $G$, by letting some of the eigenvalues of $\langle\phi\rangle$ to coincide, as is sometimes done in the literature. To do so would introduce fictitious degrees of freedom corresponding to massless, infinitely extended "solitons". In this limit all fields tend to constant values and so in fact these are not solitons at all. ${ }^{2}$ Indeed, in the case $G=S U(N)$, such "massless monopoles" do not represent any topological invariant as the fundamental group of any restored $S U(N)$ is trivial.

It is hardly possible to overemphasize the importance of the fact [4, 6, 19] that nonabelian monopoles, if they exist quantum mechanically, transform as irreducible multiplets of the dual group $\tilde{H}$, not under $H$ itself. Monopoles transforming under the dual group evade the "no-go" theorem of [10] which is a topological obstruction to the existence of monopoles transforming under the original group. The distinction between monopoles transforming under the original versus the dual group is particularly evident in the cases of $U S p(2 N)$ or $S O(2 N+1)$ gauge theories which will be considered below. For instance, in the system with spontaneous symmetry breaking, $U S p(2 N+2) \rightarrow U S p(2 N) \times U(1)$, we find (see Section 5, Appendix A that the semi-classical (hence candidate) nonabelian monopoles form a degenerate $(2 N+1)$ plet. While there are no $(2 N+1)$-dimensional representations of $H=U S p(2 N)$, the fundamental representation of the dual group $\tilde{H}=S O(2 N+1)$ has precisely the desired dimension. Analogously, in the system with gauge symmetry breaking $S O(2 N+3) \rightarrow S O(2 N+1) \times U(1)$ we find $2 N$ degenerate monopoles. Again this is the right multiplicity for $\tilde{H}=U S p(2 N)$, the group dual to $S O(2 N+1)$.

\footnotetext{
${ }^{2}$ This is analogous to what would happen to the 't Hooft - Polyakov monopole of the spontaneously broken $S U(2) \stackrel{v}{\longrightarrow} U(1)$ theory, if one were to apply the semi-classical formulae nävely in the limit $v \rightarrow 0$.
} 
The theorem of [10 however does have an implication. It tells us that the gauge symmetry of the system in the presence of nonabelian monopoles is not a direct product

$$
H \otimes \tilde{H}
$$

as sometimes suggested, but rather $\tilde{H}$ (or something else, if the physical degrees of freedom relevant is a dyon). ${ }^{3}$ The doubling of the gauge symmetry does not take place.

The strongest evidence so far that nonabelian monopoles do exist quantum mechanically and can even become dominant degrees of freedom in the infrared, comes from the $\mathcal{N}=2$ theories of Refs. [16]-[19]. For instance, in the so-called $r$-vacua $\left(r<n_{f} / 2\right)$ with an effective $S U(r) \times U(1)^{N-r+1}$ gauge group in $\mathcal{N}=2, S U(N+1)$ gauge theories, a set of magnetic particles, some in the fundamental representation of $S U(r)$, and some others singlets of $S U(r)$, appear as light, low-energy effective degrees of freedom. Their charge structure is identical to those found for the BPS semiclassical monopoles of minimal mass [19]. ${ }^{4}$ When an adjoint mass perturbation breaking supersymmetry from $\mathcal{N}=2$ to $\mathcal{N}=1$ is added, these nonabelian monopoles condense and give rise to dual superconductors (confinement) of nonabelian type, together with flavor symmetry breaking.

Identical sets of nonabelian monopoles appear in the $r$-vacua of $\mathcal{N}=2 S O(N)$ and $U S p(2 N)$ theories [17, 18], with nonzero bare quark masses. ${ }^{5}$ Again, a detailed quantum analysis shows the presence (and the crucial role) of monopoles appearing from the breaking

$$
S O(2 N), S O(2 N+1) \text { or } U S p(2 N) \rightarrow S U(r) \times U(1)^{N-r+1}, \quad r<\frac{n_{f}}{2}
$$

with the same charges as the semiclassical Goddard-Olive-Nuyts-Weinberg monopoles, but massless and playing the role of the order parameters of confinement.

\footnotetext{
${ }^{3} \mathrm{~A}$ similar conclusion is reached by Bais and Schroers [5] who did a careful analysis of surviving generators for $S U(N)$ theories.

${ }^{4}$ In fact, the monopoles can acquire flavor quantum numbers due to fermion zero modes in antisymmetric representations of $S U\left(N_{f}\right)$ [33, 19, and the "dual quarks" in the effective Lagrangian [16] at those points on the moduli space can naturally be in the fundamental representation of $S U\left(N_{f}\right)$. This makes the identification of "dual quarks" at these " $r$-vacua" with non-abelian monopoles possible. Why this particular flavor representation remains, however, is a dynamical question.

${ }^{5}$ As noted in [31] and in [17, this fact is related to the universality of these SCFTs appearing at the related singularities of the $\mathcal{N}=2$ theories [32].
} 
These examples illustrate very clearly the crucial role played by the massless flavors in the quantum theory. Without massless flavors the systems go through dynamical (gauge) symmetry breaking and the nonabelian gauge symmetry of the monopoles is destroyed.

In the presence of a sufficient number of flavors the dual $S U(r)$ gauge symmetry in (3.1) is exact in the infrared, thanks to the renormalization effects. Due to the flavor charge of the nonabelian monopoles the dual $S U(r)$ coupling constant is infrared-free

$$
b_{0}^{(d u a l)} \propto-2 r+n_{f}>0,
$$

while the original electric theory is asymptotically free:

$$
b_{0} \propto-2 N+n_{f}<0, \quad-N+2+n_{f}<0, \quad-2 N-2+n_{f}<0,
$$

(for $S U(N), S O(N)$, and $U S p(2 N)$, respectively). This is how we understand the actual occurrence of the quantum $r$ - vacua in the $\mathcal{N}=2, S U(N), S O(N)$ and $U S p(2 N)$ gauge theories, only in systems with flavors and with the value $r$ limited by $\left[\frac{n_{f}}{2}\right]$. When such a sign flip is not possible for some reason, such as in pure $\mathcal{N}=2$ $\mathrm{SYM}$ or in generic vacua of $\mathcal{N}=2$ theories, dynamical abelianization is expected to and indeed does take place.

We are thus led to draw the following conclusion. For systems with a symmetry breaking $G \rightarrow H$, the general criterion for the persistence of nonabelian monopoles in the quantum theory is that the system be such that it produces, upon symmetry breaking, a sufficient number of massless flavors carrying charges in $H$, so as to protect the latter group from becoming strongly-coupled in the infrared and from dynamically breaking itself.

Let us illustrate this for different cases considered in this paper. A detailed and concrete discussion was given in Section 2 for the system $S U(N+1) \rightarrow S U(N) \times U(1)$, which easily generalizes to a more general breaking patterns $S U(N) \rightarrow S U(r) \times$ $U(1)^{N-r+1}$.

The cases $U S p(2 N) \rightarrow S U(r) \times U(1)^{N-r+1}$ is dealt with also quite straightforwardly (see Sec. (3.2) of Ref. [17]). By embedding the system in the $\mathcal{N}=2$ context, all the $n_{f}$ hypermultiplets can be given an equal nonzero bare mass, and the breaking is achieved by the adjoint scalar VEV of the form,

$$
\phi=\frac{1}{\sqrt{2}} \operatorname{diag}\left(i m_{1}, i m_{2}, \ldots, i m_{r}, 0, \ldots,-i m_{1},-i m_{2}, \ldots-i m_{r}, 0, \ldots\right) ;
$$


where $m_{i} \rightarrow m$. The condition $r>\frac{n_{f}}{2}$ guarantees that the subgroup $S U(r)$ survives in the infrared; this last condition is clearly compatible with the asymptotic freedom of the original $U S p(2 N)$ theory $\left(n_{f}<2 N+2\right){ }^{6}$

In order to have the breaking $U S p(2 N+2) \rightarrow U S p(2 N) \times U(1)$ (or a smaller $U S p$ factor), a different setting is needed. We give only one of the hypermultiplets a large bare mass $m$, and cancel it by

$$
\phi=\frac{1}{\sqrt{2}} \operatorname{diag}(0, \ldots, 0, i m ; 0, \ldots, 0,-i m)
$$

so as to satisfy the vacuum equations. The unbroken $U S p(2 N)$ survives in the infrared if $n_{f}-1 \geq 2 N+2$ while the original $U S p(2 N+2)$ is asymptotically free for $n_{f}<$ $2 N+4$. So in this case the only possible value is $n_{f}=2 N+3$; for a smaller $U S p$ factor the condition is less severe.

In the case of the symmetry breaking, $S O(2 N+2) \rightarrow S O(2 N) \times U(1)$, we again embed the system in a $\mathcal{N}=2$ theory with $n_{f}$ hypermultiplets of which one has a large bare mass $m$, while others have none. When the adjoint scalar VEV is of an appropriate form (Eq.(5.1) below, with $v=i m / \sqrt{2}$ ), the $S O(2 N)$ components of the other $n_{f}-1$ hypermultiplets remain massless. Thus for $n_{f}-1 \geq 2 N-2$ (which is compatible with the requirement that the original theory is asymptotically free, i.e., $\left.n_{f}<2 N\right)$ the unbroken group $S O(2 N)$ is non asymptotically free. For more general patterns of breaking, $S O(2 N+2) \rightarrow S O(2 r) \times U(1)^{N-r+1}$, it is even easier to arrange the $\mathcal{N}=2$ system so that the unbroken $S O(2 r)$ is non-asymptotically free (Eq. (3.2) vs Eq. (3.3) ).

In the case of the diagonal breaking $S O(2 N) \rightarrow U(N)$ considered also below, we find that the standard embedding the system in the $\mathcal{N}=2$ version of the theory, with the adjoint VEV of the form (5.24) with $v=m$, is not sufficient to guarantee the unbroken group $U(N)$ to remain so in the infrared. With the standard $\mathcal{N}=$ 2 embedding the number of the flavors in the vector representation is limited to $n_{f}<2 N-2$, from the requirement that the original gauge group is asymptotically free. But then the "unbroken" $S U(N)$ theory necessarily grows strong in the infrared

\footnotetext{
${ }^{6}$ Of course, one could drop this requirement and work with a larger number of flavors, so that the unbroken group $H$ is always non aymptotically free, if one is satisfied with a semiclassical analysis $(m \gg \Lambda)$. The point is that interesting things - such as light nonabelian monopoles - happen when the underlying theory becomes strongly coupled in the infrared, and to study such a system one must tune the bare masses $m$ to $O(\Lambda)$ or even to zero.
} 
and breaks itself to an abelian group $U(1)^{N-1}$. Thus in order to discuss quantum nonabelian monopoles in a second rank tensor representation of $S U(N)$ one must embed the system either in a $\mathcal{N}=4$ context, or to consider different matter content.

No such problem arises for the system with $S O(2 N) \rightarrow S U(r) \times U(1)^{N-r+1}$, with $r<N$, where semiclassical monopoles in the fundamental as well as in the second rank tensor representations appear. As long as $r<\frac{n_{f}}{2}, n_{f}<2 N-2$, one easily accomodates the asymptotic freedom of the original theory with infrared freedom of the subgroup $S U(r)$, by giving the equal bare masses to all the hypermultiplets and by choosing the adjoint scalar VEVs of the form (5.24) with $v=m$. In the region $m \sim \Lambda$, the semiclassical reasoning only does not tell which nonabelian monopoles survive in the infrared; the quantum analysis of [18] shows that it is the monopoles in the fundamental representation of the dual $S U(r)$ that becomes light due to the quantum effects.

The situation is a little similar, in the case of $\mathcal{N}=2$ theories with symmetry breaking, $S U(N) \rightarrow S U(r) \times S U(s) \times U(1)^{N-r-s+1}$. For $r$ and $s$ not too large, the condition for the persistence of quantum monopoles $f_{1} \geq 2 r, f_{2} \geq 2 s$ can be satisfied in an appropriate vacuum, where $f_{1}\left(f_{2}\right)$ is the number of flavors with bare mass $m_{1}$ $\left(m_{2}\right), f_{1}+f_{2}<n_{f}$. All other $n_{f}-f_{1}-f_{2}$ flavors must be given unequal bare masses $m_{i}$. Again, the case of maximal nonabelian factors $(r+s=N)$ discussed in the subsection 4.2 is special, in that the only semiclassical monopoles are in the $\left(\underline{r}, \underline{s}^{*}\right)$ representation in this case, and in that the requirement of persistence of unbroken $S U(r) \times S U(s)$ group, is not compatible with the asymptotic freedom of the fundamental $S U(N)$ interactions, in the standard $\mathcal{N}=2$ setting.

As we have already mentioned nonabelian monopoles also appear in the nontrivial superconformal vacua of $\mathcal{N}=2$ models. Examples include the limiting case $\left(r=\left[\frac{n_{f}}{2}\right]\right)$ of the so-called $r$ vacua

$$
S U(N+1), S O(2 N), S O(2 N+1) \text { or } U S p(2 N) \rightarrow S U\left(\frac{n_{f}}{2}\right) \times U(1)^{N-\frac{n_{f}}{2}+1}
$$

with equal nonzero bare quark masses, as well as all of the confining vacua of $S O(N)$, and $U S p(2 N)$ theories with vanishing bare quark masses [17, 18]. The physics in these cases, although perhaps the most interesting from the point of view of understanding QCD, is complicated by the simultaneous presence of relatively nonlocal massless monopoles and dyons. No local effective Lagrangian description is available, in general. To the best of our knowledge, the study of the physical properties of this kind of systems is still at an exploratory stage (see for example [20], however). 


\section{Monopole Spectra from Homotopy Groups}

In this section we attempt to classify topologically inequivalent monopole configurations, leaving the explicit construction of the monopoles to Section 5 and Appendix A. A monopole is topologically stable whenever a 2-sphere surrounding the monopole supports a nontrivial gauge bundle. The topology of this bundle is determined entirely by the homotopy type $\pi_{1}(H)$ of the transition function $S^{1} \longrightarrow H$ on the equatorial circle. This is not the only data that specifies a monopole configuration, in addition there is an adjoint Higgs field, which is valued in the original gauge group $G$. Equivalence classes of the Higgs field under the unbroken $H$ gauge symmetry give a $G / H$-valued function. The asymptotic value of the Higgs field on the 2-sphere at infinity gives a map $S^{2} \longrightarrow G / H$ that represents a class $\pi_{2}(G / H)$. Thus any configuration seems to be characterized by pair of topological invariants: $\pi_{1}(H)$ and $\pi_{2}(G / H)$.

Here we are interested in only the subset of monopoles which are finite-energy, regular field configurations of 't Hooft-Polyakov type. In particular cases, e.g., when the model is embedded in a $\mathcal{N}=2$ theory these monopoles are BPS: they satisfy the linear, nonabelian Bogomolny equations. Otherwise, they are solutions only to quadratic Yang-Mills-scalar coupled field equations. These equations allow us to determine up to a constant the profile of the Higgs field from that of the gauge field and vice versa. This means that if we are given only, for example, a class in $\pi_{1}(H)$ that describes the gauge field configuration then there is at most one class in $\pi_{2}(G / H)$ that describes the Higgs VEV. Thus each distinct BPS monopole ${ }^{7}$ is entirely characterized by a class in $\pi_{1}(H)$. However there may be classes in $\pi_{1}(H)$ that do not correspond to any class in $\pi_{2}(G / H)$ and therefore do not correspond to any BPS monopole. This means that in general BPS monopoles are classified by only a subset of $\pi_{1}(H)$, the subset that correspond to elements of $\pi_{2}(G / H)$.

So to classify BPS monopoles we need to know what the correspondence is between gauge field configurations $\pi_{1}(H)$ and Higgs field configurations $\pi_{2}(G / H)$. This correspondence is determined by the nonabelian Bogomolny equations, but we claim that this is the same correspondence that arises from the long exact sequence for

\footnotetext{
${ }^{7}$ In the following we shall use the term, "BPS monopoles", having in mind the particularly elegant cases such as $\mathcal{N}=2$ or $\mathcal{N}=4$ theories, but the whole discussion is valid for more general nonsingular solitonlike monopoles.
} 
homotopy groups of fibrations

$$
\cdots \rightarrow \pi_{n}(H) \rightarrow \pi_{n}(G) \rightarrow \pi_{n}(G / H) \rightarrow \pi_{n-1}(H) \rightarrow \cdots
$$

Using Poincaré's theorem $\left(\pi_{2}(G)=0\right.$ for any compact Lie group) and restricting our attention to cases in which $G / H$ is simply connected we find the short exact sequence

$$
0 \rightarrow \pi_{2}(G / H) \stackrel{f}{\rightarrow} \pi_{1}(H) \rightarrow \pi_{1}(G) \rightarrow 0 .
$$

The desired correspondence is then the above inclusion $f$ of $\pi_{2}(G / H)$ into $\pi_{1}(H)$, which implies that every class in $\pi_{2}(G / H)$ corresponds to a BPS monopole. On the other hand if $G$ is not simply connected then only one element of $\pi_{1}(H)$ of every $\left|\pi_{1}(G)\right|$ (the cardinality of $\pi_{1}(G)$ ) elements corresponds to a nonsingular monopole. ${ }^{8}$ The others correspond to configurations containing singular Dirac-like monopoles in the original group $G$, which are enumerated by $\pi_{1}(G)$ and do not involve the Higgs field.

Our problem is then reduced to the problem of finding $\pi_{2}(G / H)$. The above short homotopy sequence yields the relation

$$
\pi_{1}(G)=\pi_{1}(H) / \pi_{2}(G / H)
$$

This formula, together with an embedding of $H$ into $G$ will always allow us to determine $\pi_{2}(G / H)$ and thus to enumerate the nonabelian monopoles. As every monopole configuration corresponds to a class in $\pi_{1}(H)$ that is represented by the transition function of the gauge bundle, this correspondence will automatically allow us to find the transition functions in the cases below and thus to determine the topologies of the gauge bundles. For this purpose we will make extensive use of Table 1 which lists the centers and fundamental groups of the relevant semi-simple Lie groups.

\section{1 $S U(N+1) \rightarrow S U(N) \times U(1)$}

Let us start by revisiting the case of an $S U(N+1)$ theory spontaneously broken to $H=U(N)=S U(N) \times U(1) / \mathbb{Z}_{N}$. The coset space is nothing but the projective

\footnotetext{
${ }^{8}$ Alternately, "the condition for a nonsingular monopole is that the topological charge is in the kernel of the mapping $\pi_{1}(H) \rightarrow \pi_{1}(G)$ " [7]. While our perspective makes use of the Bogomolny equations to identify the BPS monopoles, this perspective uses the topology of the gauge bundle to identify the nonsingular monopoles. In view of Eq.4.3 these two classification schemes yield identical monopole spectra.
} 


\begin{tabular}{|ccc|}
\hline $\mathbf{G}$ & Center & $\pi_{1}(G)$ \\
\hline$S U(N+1)$ & $\mathbb{Z}_{N+1}$ & $\mathbf{0}$ \\
\hline$U S p(2 N)$ & $\mathbb{Z}_{2}$ & $\mathbf{0}$ \\
\hline$S O(2 N+1)$ & $\mathbf{1}$ & $\mathbb{Z}_{2}$ \\
\hline $\operatorname{Spin}(2 N+1)$ & $\mathbb{Z}_{2}$ & $\mathbf{0}$ \\
\hline$S O(2 N)$ & $\mathbb{Z}_{2}$ & $\mathbb{Z}_{2}$ \\
\hline $\operatorname{Spin}(4 N)$ & $\mathbb{Z}_{2} \times \mathbb{Z}_{2}$ & $\mathbf{0}$ \\
\hline $\operatorname{Spin}(4 N+2)$ & $\mathbb{Z}_{4}$ & $\mathbf{0}$ \\
\hline
\end{tabular}

Table 1: Centers and the fundamental groups of various compact Lie groups

sphere $\mathbb{C} P^{N}=U(N+1) /(U(N) \times U(1))$. By abuse of notation we will sometimes omit the quotient, as we have done in the title of this subsection. This discussion can be easily generalized to, for example, $S U(N+1) \rightarrow S U(r) \times U(1)^{N+1-r}$.

The generator of the unbroken $U(1)$ gauge group is

$$
Q=\operatorname{diag}(1, \cdots, 1,-N)
$$

Meanwhile the $S U(N)$ consists of $N+1$ by $N+1$ matrices whose top left $N$ by $N$ submatrix is in $S U(N)$ and whose remaining row and column consist of all zeros and a single one in the lower-right corner. The $N$ elements

$$
e^{2 \pi i Q / N}=\operatorname{diag}\left(e^{2 \pi i / N}, \cdots, e^{2 \pi i / N}, 1\right)
$$

appearing in $U(1)$ are also the $\mathbb{Z}_{N}$ center of the unbroken $S U(N)$ group. This is the reason for the $\mathbb{Z}_{N}$ quotient in the definition of $H$.

$G=S U(N+1)$ is simply connected, and so $\pi_{2}(S U(N+1) / U(N))$ is equal to $\pi_{1}(U(N))$, implying that either one classifies nonsingular (BPS in a supersymmetric embedding) monopoles. To calculate $\pi_{1}(U(N))$ we use the exact sequence

$$
\begin{aligned}
& 0=\pi_{1}\left(\mathbb{Z}_{N}\right) \rightarrow \pi_{1}(S U(N) \times U(1)) \rightarrow \pi_{1}(U(N)) \\
& \rightarrow \pi_{0}\left(\mathbb{Z}_{N}\right) \rightarrow \pi_{0}\left((S U(N) \times U(1)) / \mathbb{Z}_{N}\right)=0 .
\end{aligned}
$$

By the Kunneth formula $\pi_{1}(S U(N) \times U(1))=\pi_{1}(U(1))=\mathbb{Z}$, while $\pi_{0}\left(\mathbb{Z}_{N}\right)=\mathbb{Z}_{N}$. This short exact sequence then yields the equality

$$
\mathbb{Z}_{N}=\pi_{1}(U(N)) / \mathbb{Z}
$$


implying that $\pi_{1}(U(N))$, the group that classifies monopoles, is isomorphic to $\mathbb{Z}$ possibly times a finite cyclic group whose generator is a loop from the identity to the generator of $\mathbb{Z}_{N}$. In fact there is no additional finite cyclic group: the new generator is just $1 / N$ of the old generator of the $\pi_{1}(U(1))$ (See Fig 1 )

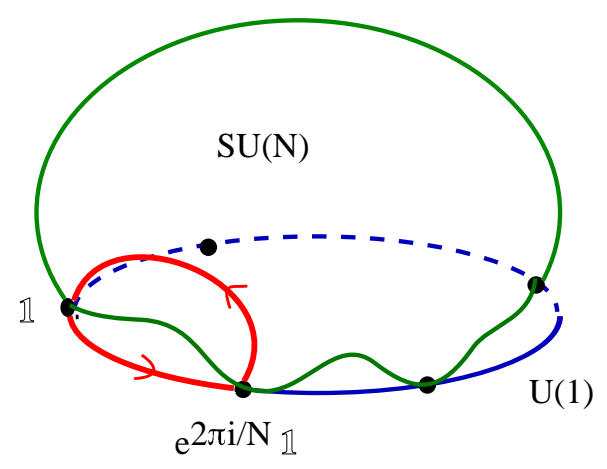

Figure 1: The smallest closed loop in $U(N)$

This point is easy to understand. In $S U(N) \times U(1)$, the only non-contractible loops are those that go around the $U(1)$ an integral number of times. However within $H=U(N)$, there is a loop that goes inside $S U(N)$ from the identity element to the $\mathbb{Z}_{N}$ center, which can also be regarded as an element of $U(1)$, and then comes back to the identity inside of the $U(1)$. If we travel around this loop $N$ times then we have gone around the $U(1)$ one full time, and we have also made a loop in $S U(N)$. $S U(N)$ is simply-connected, and so we can deform away the loop that we have made in $S U(N)$, and conclude that $N$ times the generator of $\pi_{1}(U(N))$ is the generator of $\pi_{1}(S U(N) \times U(1))=\pi_{1}(U(1))$.

We have then learned that $\pi_{1}(U(N))$ is isomorphic to $\mathbb{Z}$, yielding precisely one family of BPS monopoles.

The fact (Eq.(2.18) ) that the $U(1)$ magnetic charge of the nonabelian monopole is $1 / N$ in units of the Dirac quantum for the product theory is due to this $N$ to 1 embedding of $\pi_{1}(S U(N) \times U(1))$ into $\pi_{1}(U(N))$. Our monopole has a smaller magnetic charge than any monopole that could exist in a theory with only this $U(1)$ because we have a smaller loop at our disposal with which to construct the transition function of the gauge bundle. Equivalently the minimal electric charge with respect to the $U(1)$ in the $S U(N+1) \rightarrow U(N)$ theory is $N$ times the Dirac quantum for the $U(1)$ due to the constraint that all matter must descend from representations of the 
original $S U(N+1)$.

To make this discussion more concrete, let us present the gauge transformation (transition function) along the equator of an $S^{2}$ that surrounds a monopole [2]. The coordinate along the equator is the azimuth $\phi$, and we have the $S U(N)$ element

$$
\operatorname{diag}\left(e^{-i \phi(N-1) / N}, e^{i \phi / N}, \cdots, e^{i \phi / N}, 1\right)
$$

and the $U(1)$ element

$$
\operatorname{diag}\left(e^{-i \phi / N}, \cdots, e^{-i \phi / N}, e^{-i \phi / N}, e^{i \phi}\right) .
$$

The action of these elements on matter in the fundamental representation is just the product of the two,

$$
\operatorname{diag}\left(e^{-i \phi}, 1, \cdots, 1, e^{i \phi}\right) .
$$

It clearly is single-valued when one comes back around the equator. In fact it represents the generator of $\pi_{1}(U(N))$, as it is just the loop described above in which one travels to a root of unity in the $U(1)$ and then returns to the identity inside the $S U(N)$. Thus we have found the transition function for a monopole configuration with a single unit of charge. This monopole is nothing but the embedding of the usual $S U(2)$ monopole between the first unbroken color $i=1$ and the abelian part $i=N+1$, i.e. the $(1,0, \cdots)$ - monopole.

One might be tempted to believe that the choice of unbroken color, that is the choice of loop in $S U(N)$, leads to $N$ independent monopole solutions. However the transition functions corresponding to all possible choices are homotopic to each other, and so the topology of the bundle is independent of this choice. The dual gauge transformations under which our monopoles transform, being continuous, necessarily preserve the topology of the gauge bundle. Thus we cannot find the dimensions of the representations inhabited by monopoles by merely counting distinct bundles nor by counting homotopically inequivalent transition functions because all transition functions in a given multiplet are homotopic.

The main point is that nonabelian monopoles transform under the dual gauge group, which in this case is another copy of the original unbroken gauge group $H=U(N)$. To determine what representation of $U(N)$ acts on our monopoles, we decompose the monopoles in terms of the $U(1)$ and $S U(N)$ actions. We have seen that the $U(1)$ charge is the minimum possible value, $1 / N$, and so we need only find the representation of $S U(N)$ under which the monopoles transform. 
We claim that in general monopoles transform under some subset of the $k$ th symmetric tensor representation of the dual gauge group if their transition function represents the element $k \in \mathbb{Z}$ in $H$. In general the subset and therefore the representation is not determined by the topology, ${ }^{9}$ but in this case we have seen that $k=1$ and so our monopoles transform in a rank one tensor representation of $S U(N)$. There are only two such representations, the fundamental and antifundamental representations. Thus we may conclude that the lightest mass monopoles transform in the (anti-)fundamental representation of $S U(N)$ and have charge $1 / N$ under $U(1)$, in short, they transform under the $N$ of $U(N)$.

This construction is consistent with the general construction of [4, 6], summarized in Appendix A according to which each nonabelian monopole is associated with a root vector corresponding to a broken generator, and their properties are determined by the dual of such root, $\alpha^{*} \equiv \alpha / \alpha \cdot \alpha$. The monopoles arising from the breaking $S U(N+1) \rightarrow S U(N) \times U(1)$ transform in the (anti-)fundamental representation of the dual $S U(N)$.

\section{$4.2 \quad S U(N+M) \rightarrow S U(N) \times S U(M) \times U(1)$}

This is a simple generalization of the previous case, yet is it interesting that the unbroken group involves two non-abelian groups. The coset group is actually $H=$ $(S U(N) \times S U(M) \times U(1)) / \mathbb{Z}_{k}$ where $k$ is the least common multiple $k=\operatorname{LCM}(N, M)$. The coset space is nothing but the Grassmannian manifold $G_{N, N+M}(\mathbb{C})=U(N+$ $M) /(U(N) \times U(M))$. The homotopy groups are obtained by

$$
0=\pi_{2}(H) \rightarrow \pi_{2}(G / H) \rightarrow \pi_{1}(H)=\mathbb{Z} \rightarrow \pi_{1}(G)=0 .
$$

Therefore, the monopoles are classified by $\pi_{2}(G / H)=\mathbb{Z}$.

The generator of $U(1)$ is

$$
Q=\left(\begin{array}{l|l}
\frac{1}{N} \mathbf{1}_{N} & \\
\hline & -\frac{1}{M} \mathbf{1}_{M}
\end{array}\right) .
$$

It is clear that $k Q$ has integer eigenvalues by definition, and hence $e^{2 \pi i Q}$ is the generator of $\mathbb{Z}_{k}$. The transition function along the equator is given by $e^{i Q \phi}$ for the $U(1)$,

\footnotetext{
${ }^{9}$ While the representation is not determined from the gauge bundle's topology alone, it is determined by the Lie algebra-valued curvature 2 -form of the bundle, which is the monopoles magnetic flux. It transforms under the same representation as the monopole.
} 
which is canceled by the center elements of $S U(N)$ and $S U(M)$.

Following the same analysis as in the previous section, the smallest monopole transforms as $(N, \bar{M})_{1 / k}$ under $S U(N) \times S U(M) \times U(1)$.

\section{$4.3 \quad S O(N+2) \rightarrow S O(N) \times U(1)$}

As always, the BPS nonabelian monopoles are classified by $\pi_{2}(G / H)=\pi_{2}(S O(N+$ $2) / S O(N) \times U(1))$. This homotopy group may be evaluated using the short exact sequence

$$
\begin{aligned}
& 0=\pi_{2}(S O(N+2)) \rightarrow \pi_{2}(S O(N+2) / S O(N) \times U(1)) \rightarrow \\
& \stackrel{f}{\rightarrow} \pi_{1}(S O(N) \times U(1))=\mathbb{Z} \times \mathbb{Z}_{2} \stackrel{g}{\rightarrow} \pi_{1}(S O(N+2))=\mathbb{Z}_{2} \rightarrow 0 .
\end{aligned}
$$

It follows that $\pi_{2}(S O(N+2) / S O(N) \times U(1))=\mathbb{Z}$, and so again we find precisely one family of BPS monopoles.

To find the transition function we use the above map $f$, whose image is the set of homotopy classes of transition functions of the gauge bundle. The surjectivity of $g$ implies that the image of $f$ consists of only half of the elements of $\pi_{1}(H)$

$$
f\left(\pi_{2}(S O(N+2) / S O(N) \times U(1))\right)=\mathbb{Z} \subset \mathbb{Z} \times \mathbb{Z}_{2}=\pi_{1}(S O(N) \times U(1)) .
$$

The transition function representing the nontrivial element of $\mathbb{Z}_{2}$ is not in the image of $f$ and so describes a singular, Dirac-like monopole. In fact it is the usual $\mathbb{Z}_{2}$ monopole that exists in $S O$ gauge theories even when the symmetry is unbroken [2]. The transition function of the smallest BPS monopole represents the generator of $\mathbb{Z} \subset \pi_{1}(H)$ which is the image under $f$ of the generator of $\pi_{2}(G / H)$.

This monopole has a full unit of charge with respect to that of a pure $U(1)$ monopole because, unlike the previous case, the $U(1)$ is not quotiented and so the transition function $S^{1} \rightarrow H$ needs to wrap the entire $U(1)$ in order to be single-valued. Indeed we may explicitly construct the transition function around the equator as follows. We can make the $2 \pi$ rotation in $S O(N)$ along any direction, and with no loss of generality we take consider the rotation in the $(j, k)$ plane. The unbroken $U(1)$ group is also a rotation but in the $(N+1, N+2)$ plane. In other words, all the action is within an $S O(4)=S U(2) \times S U(2)$ subgroup of $S O(N+2)$, and the smallest monopole is nothing but the monopole in one of the $S U(2)$ factors of $S O(4)$. 
There are ${ }_{N} C_{2}=(N-1) N / 2$ choices of $(j, k)$ plane in $S O(N)$. Apparently, there are $(N-1) N / 2$ independent monopole solutions. However this argument is too fast as every choice of $(j, k)$ leads to a homotopically equivalent transition function and so a topologically equivalent bundle. Instead we apply the above proposal that monopoles transform in a $k$ th tensor representation of the dual gauge group if their transition function represents the element $k$ in the free part of $\pi_{1}(H)$. We have seen that for the lightest monopoles, constructed above, $k=1$ and so these monopoles transform in the fundamental representation of the dual $S O(N)$ (if $N$ is even) or $S p(N-1)$ (if $N$ is odd) gauge group. We will find the same result in the next section when we explicitly construct the monopole solutions.

\section{4 $U S p(2 N+2) \rightarrow U S p(2 N) \times U(1)$}

This example is simpler than the previous as USp groups are simply connected. The usual short exact sequence then provides an isomorphism

$$
\pi_{2}\left(\frac{U S p(2 N+2)}{U S p(2 N) \times U(1)}\right) \cong \pi_{1}(U S p(2 N) \times U(1)) \cong \mathbb{Z} .
$$

Again we find a single family of BPS monopoles. However this time the simplyconnectedness of $U S p$ ensures that there are no singular monopoles and so every transition function in $\pi_{1}(H)$ yields a desired gauge bundle. The mass is determined by the $\pi_{1}(U(1))$ contribution to the transition function, and so the lightest BPS monopoles will be those corresponding to the degree one map from the equator to $U(1)$.

Again the fact that this map is the generator of the fundamental group implies that these monopoles transform in the fundamental representation of the dual group, which is $S O(2 N+1)$. The appearance of a $(2 N+1)$-dimensional representation of monopoles constructed using $U S p(2 N)$ may appear surprising. We will see when we explicitly construct these monopoles in the next section that in addition to the $2 \mathrm{~N}$ monopoles constructed from the $N S U(2)$ 's in $U S p(2 N)$ whose Cartan generators generate the Cartan subalgebra of $U S p(2 N)$, there is a $(2 N+1)$ th monopole that is the standard 't Hooft Polyakov monopole corresponding to the extra unbroken $U(1)$. In the full quantum theory these apparently very different types of monopoles are in fact degenerate and gauge equivalent under the dual $S O(2 N+1)$. This nicely illustrates the importance of distinguishing between the unbroken gauge symmetry and its dual. 


\section{5 $S O(2 N) \rightarrow U(N)$}

This breaking is possible, for example, in an $\mathcal{N}=2 S O(2 N)$ gauge theory when the quarks in the vector representation have a degenerate mass which is precisely canceled by the adjoint VEV. As this mass approaches zero the full $S O(2 N)$ symmetry is restored.

The homotopy exact sequence is now

$$
\begin{aligned}
0= & \pi_{2}(S O(2 N)) \rightarrow \pi_{2}(S O(2 N) / U(N)) \stackrel{f}{\rightarrow} \pi_{1}(U(N))=\mathbb{Z} \\
& \stackrel{g}{\rightarrow} \pi_{1}(S O(2 N))=\mathbb{Z}_{2} \rightarrow 0=\pi_{1}(S O(2 N) / U(N)) .
\end{aligned}
$$

To see that $\pi_{1}(S O(2 N) / U(N))$ vanishes, we will use the fact that our monopoles are all embeddings of the standard 't Hooft Polyakov monopoles in broken $S U(2)$ gauge theories. Thus it suffices to consider the topology of a $S O(4) \rightarrow U(2)$ in which the monopole is embedded, and so we need only show that $\pi_{1}(S O(4) / U(2))=0$. $S O(4)$ is the twisted product of two three-spheres, and the action of $S U(2)$ on each 3-sphere is given by the identification of the 3 -sphere with $S U(2)$ and the group multiplication in $S U(2)$. We may then quotient $S O(4)$ by $U(2)$ in two steps, first we quotient by the action of $S U(2)$ and then by the action of $U(1)$. As the $S U(2)$ acts diagonally on the two 3-spheres, $S O(4) / S U(2)$ is $S U(2) / Z_{2}=S O(3)$, which is topologically a circle bundle over a 2 -sphere with Chern class equal to two. The quotient of the total space of this bundle by its fiber, a circle, is just the base space of the bundle, which is a 2-sphere. The 2-sphere is simply-connected and so $\pi_{1}(S O(4) / U(2))=\pi_{1}\left(S^{2}\right)=0$ as claimed.

The surjectivity of $g: \mathbb{Z} \rightarrow \mathbb{Z}_{2}$ implies that the image of $f$ is the set of even integers. $f$ must be one to one and so its domain, $\pi_{2}(S O(2 N) / U(N))$ must be the set of integers. This is consistent with the above topological description of $S O(2 N) / U(N)$ as the 2-sphere, as $\pi_{2}(S O(2 N) / U(N))=\pi_{2}\left(S^{2}\right)=\mathbb{Z}$. Every element of $\pi_{1}(U(N))=$ $\mathbb{Z}$ describes a gauge bundle, but as only the even elements are in the image of $f$, only the even elements describe BPS monopoles. The odd elements are transition functions of singular configurations that include the singular $\mathbb{Z}_{2}$ monopoles of the original $S O$ gauge theory. Due to the $N$ to 1 embedding of $U(1)$ in $U(N)$, the lowest possible monopole charge is $1 / N$ of the Dirac quantum corresponding to the element $1 \in \pi_{1}(U(N))$. Therefore the minimum possible charge of a BPS monopole is $2 / N$, corresponding to the smallest even element $2 \in \pi_{1}(U(N))$. 
Using our proposed relation between elements of the fundamental group and representations we then conclude that, in addition to the above $U(1)$ charge these monopoles transform under some second rank tensor representation of the dual $S U(N)$ gauge symmetry. However topological considerations alone do not allow us to determine which second rank tensor representation yields a BPS monopole. The answer to this question will need to await the next section, in which the monopoles are explicitly constructed.

\section{6 $S O(2 N+1) \rightarrow U(N)$}

The analysis in this case proceeds identically to the preceding case. The verification that $\pi_{1}(S O(2 N+1) / U(N))=0$ again reduces to a computation of $S O(4) / U(2)$ using the fact that the monopole solution is the embedding of an $S U(2)$ monopole (or more accurately perhaps an $S O(3)$ monopole) into some $S O(4) \rightarrow U(2)$ subsector. And so we again conclude that there are BPS nonabelian monopoles with charge $2 / N$ under the unbroken $U(1)$ that transform under an unknown second rank tensor representation of the dual $S U(N)$. The fact that the topology does not tell us which second rank tensor to use is highlighted by the fact that, as we will see, despite the identical homotopy groups in the two examples the corresponding monopoles transform under different second rank tensor representations of the dual $S U(N)$.

\section{$4.7 \quad U S p(2 N) \rightarrow U(N)$}

This breaking is possible, for example, in an $\mathcal{N}=2 U S p(2 N)$ theory when the $N$ quarks have a degenerate mass which is precisely canceled by the adjoint VEV. The fundamental representation of $U S p(2 N)$ decomposes as $2 N=(N,+1) \oplus(\bar{N},-1)$. The center of $S U(N)$ can be regarded as an element of $U(1)$, and hence the quotient by $\mathbb{Z}_{N}$.

$U S p(2 N)$ is simply connected and so every transition function in $\pi_{1}(S U(N))$ is the image of some Higgs field in $\pi_{2}(U S p(2 N) / S U(N))$. Thus for every allowed charge in $\pi_{1}(S U(N))=\mathbb{Z}$ there is a solution to the Bogomolny equations yielding a BPS monopole configuration The smallest such charge, in units of the Dirac quantum of the $U(1)$, is then $1 / N$. The corresponding transition function is a representative of the generator of $\pi_{1}(U(N))$. This may be written as a product of generators of the 
fundamental groups of $S U(N)$ and $U(1)$, such as $\operatorname{diag}\left(e^{i \phi / N}, \cdots, e^{i \phi / N}, e^{-i(N-1) \phi / N}\right) \in$ $S U(N)$ and $e^{-i \phi / N} \in U(1)$ which multiply to

$$
\operatorname{diag}\left(1, \cdots, 1, e^{-i \phi}\right) \in U(N)
$$

Looking at the whole fundamental representation of $U S p(2 N)$, one can see that it is a monopole embedded in $S U(2) \simeq U S p(2) \subset U S p(2 N)$. The monopoles therefore transform as the fundamental representation of the $S U(N)$ group, as follows, for example, from the fact that the transition function represents $1 \in \pi_{1}(H)$.

\section{8 $S O(N+2 M) \rightarrow S O(N) \times U(M)$}

The breaking is possible, for example, in an $\mathcal{N}=2 S O(N+2 M)$ theory when $M$ quarks have a degenerate mass which is precisely canceled by the adjoint VEV. The fundamental representation of $S O(N+2 M)$ decomposes as $N+2 M=(N, 1) \oplus$ $(1, M) \oplus(1, \bar{M})$.

Combining analyses in Sec. 4.3, 4.5, 4.6, we find that the monopoles are classified according to $\pi_{2}\left(S O(N+2 M) /(S O(N) \times U(M))=\pi_{1}(U(M))=\mathbb{Z}\right.$. When $N$ is even, the monopoles transform under the dual $S O(N) \times U(M)$ group as $(N, M)_{1 / M} \oplus$ $\left(1, \frac{M(M-1)}{2}\right)_{2 / M}$, where the latter may decay into the pair of the former. When $N$ is odd, the monopoles transform under the dual $U S p(N-1) \times U(M)$ as $(N-1, M)_{1 / M} \oplus$ $\left(1, \frac{M(M+1)}{2}\right)_{2 / M}$, where the latter may decay into the pair of the former.

\section{$4.9 U S p(2 N+2 M) \rightarrow U S p(2 N) \times U(M)$}

The breaking is possible, for example, in an $\mathcal{N}=2 U S p(2 N+2 M)$ theory when $M$ quarks have a degenerate mass which is precisely canceled by the adjoint VEV. The fundamental representation of $U S p(2 N+2 M)$ decomposes as $N+2 M=(2 N, 1) \oplus$ $(1, M) \oplus(1, \bar{M})$.

Combining analyses in Sec. 4.4 and 4.7, we find that the monopoles are classified according to $\pi_{2}\left(U S p(2 N+2 M) /(U S p(2 N) \times U(M))=\pi_{1}(U(M))=\mathbb{Z}\right.$. The monopoles transform under the dual $S O(2 N+1) \times U(M)$ group as $(2 N+1, M)_{1 / M} \oplus$ $\left(1, \frac{M(M-1)}{2}\right)_{2 / M}$, where the latter may decay into the pair of the former. 


\section{Explicit Constructions of BPS Nonabelian Monopoles}

In this section we construct the lightest nonabelian monopole solutions by simply identifying various minimally embedded $S U(2)$ subgroups 34 which are broken to $U(1)$, and then by embedding the 't Hooft-Polyakov monopoles into this $S U(2)$ and adding a constant term to $\phi$ so that in a given spacial direction it takes the standard, prescribed form. The results agree in all cases with the general formulae given in Appendix A and with the classifications of Section 4 . The $S U(N+1)$ case has already been discussed in Section 2.1, and so below we consider the $S O(N)$ and $U S p(2 N)$ examples.

In the previous section we characterized monopoles by $\pi_{1}(H)$, whose representatives are transition functions of the gauge bundles of BPS monopoles. In this section we will find the adjoint scalar profiles, whose gauge equivalence classes represent elements of $\pi_{2}(G / H)$. The interested reader need only substitute these results into the nonabelian Bogomolny equations to find the corresponding gauge potentials.

The explicit solutions of the adjoint scalars will allow us to determine in each case the representation of the dual gauge group under which the nonabelian monopoles transform. The relevant pairs of dual groups are listed in Table 2

\begin{tabular}{rlc}
\hline$S U(N) / \mathbb{Z}_{N}$ & $\Longleftrightarrow S U(N)$ \\
$S O(2 N)$ & $\Longleftrightarrow S O(2 N)$ \\
$S O(2 N+1)$ & $\Longleftrightarrow$ & $U S p(2 N)$ \\
\hline
\end{tabular}

Table 2: Some examples of dual pairs of groups

\section{$\mathbf{5 . 1} S O(2 N+3) \rightarrow S O(2 N+1) \times U(1)$}

An adjoint scalar VEV of the form

$$
\phi=i\left(\begin{array}{ccc|cc}
0 & 0 & \ldots & 0 & 0 \\
0 & 0 & \ldots & 0 & 0 \\
\vdots & \vdots & \ddots & \vdots & \vdots \\
\hline 0 & 0 & \ldots & 0 & v \\
0 & 0 & \ldots & -v & 0
\end{array}\right)=i\left(\begin{array}{c|cc}
\mathbf{0}_{(2 N+1) \times(2 N+1)} & & \\
\hline & 0 & v \\
-v & 0
\end{array}\right)
$$


breaks an $S O(2 N+3)$ gauge symmetry to $S O(2 N+1) \times U(1)$. We will use two minimally-embedded $S U(2)$ subgroups:

$$
\begin{aligned}
& S_{1}=\frac{i}{2}\left(\begin{array}{ccc|cc}
\ddots & \vdots & \vdots & \vdots & \vdots \\
\ldots & 0 & 0 & 1 & 0 \\
\ldots & 0 & 0 & 0 & -1 \\
\hline \ldots & -1 & 0 & 0 & 0 \\
\ldots & 0 & 1 & 0 & 0
\end{array}\right), \quad S_{2}=\frac{i}{2}\left(\begin{array}{ccc|cc}
\ddots & \vdots & \vdots & \vdots & \vdots \\
\ldots & 0 & 0 & 0 & 1 \\
\ldots & 0 & 0 & 1 & 0 \\
\hline \ldots & 0 & -1 & 0 & 0 \\
\ldots & -1 & 0 & 0 & 0
\end{array}\right) \text {, } \\
& S_{3}=\frac{i}{2}\left(\begin{array}{ccc|cc}
\ddots & \vdots & \vdots & \vdots & \vdots \\
\ldots & 0 & 1 & 0 & 0 \\
\ldots & -1 & 0 & 0 & 0 \\
\hline \ldots & 0 & 0 & 0 & 1 \\
\ldots & 0 & 0 & -1 & 0
\end{array}\right) \\
& \tilde{S}_{1}=\frac{i}{2}\left(\begin{array}{ccc|cc}
\ddots & \vdots & \vdots & \vdots & \vdots \\
\ldots & 0 & 0 & -1 & 0 \\
\ldots & 0 & 0 & 0 & -1 \\
\hline \ldots & 1 & 0 & 0 & 0 \\
\ldots & 0 & 1 & 0 & 0
\end{array}\right), \quad \tilde{S}_{2}=\frac{i}{2}\left(\begin{array}{ccc|cc}
\ddots & \vdots & \vdots & \vdots & \vdots \\
\ldots & 0 & 0 & 0 & -1 \\
\ldots & 0 & 0 & 1 & 0 \\
\hline \ldots & 0 & -1 & 0 & 0 \\
\ldots & 1 & 0 & 0 & 0
\end{array}\right) \text {, } \\
& \tilde{S}_{3}=\frac{i}{2}\left(\begin{array}{ccc|cc}
\ddots & \vdots & \vdots & \vdots & \vdots \\
\ldots & 0 & -1 & 0 & 0 \\
\ldots & 1 & 0 & 0 & 0 \\
\hline \ldots & 0 & 0 & 0 & 1 \\
\ldots & 0 & 0 & -1 & 0
\end{array}\right)
\end{aligned}
$$

We may create a nonabelian monopole using the first $S U(2)$, in which case the adjoint scalar profile is:

$$
\phi=\frac{i}{2}\left(\begin{array}{ccc|cc}
\ddots & \vdots & \vdots & \vdots & \vdots \\
\ldots & 0 & -v & 0 & 0 \\
\ldots & v & 0 & 0 & 0 \\
\hline \ldots & 0 & 0 & 0 & v \\
\ldots & 0 & 0 & -v & 0
\end{array}\right)-v(\vec{S} \cdot \widehat{r}) \phi(r)
$$


or by using the second $S U(2)$ :

$$
\phi=\frac{i}{2}\left(\begin{array}{ccc|cc}
\ddots & \vdots & \vdots & \vdots & \vdots \\
\cdots & 0 & v & 0 & 0 \\
\ldots & -v & 0 & 0 & 0 \\
\hline \ldots & 0 & 0 & 0 & v \\
\ldots & 0 & 0 & -v & 0
\end{array}\right)-v(\overrightarrow{\tilde{S}} \cdot \widehat{r}) \phi(r) .
$$

The two types of monopole have the same mass:

$$
M=\frac{4 \pi v}{g} .
$$

As there are $(2 N+1)$ rows of broken group generators, one can construct in this way $2 N$ independent minimally-embedded monopoles. For the last row one is forced to use the non-minimal $S O(3)$ embedding:

$$
\begin{gathered}
S_{1}=i\left(\begin{array}{cc|cc}
\ddots & \vdots & \vdots & \vdots \\
\ldots & 0 & -1 & 0 \\
\hline \ldots & 1 & 0 & 0 \\
\ldots & 0 & 0 & 0
\end{array}\right), \quad S_{2}=i\left(\begin{array}{cc|cc}
\ddots & \vdots & \vdots & \vdots \\
\ldots & 0 & 0 & -1 \\
\hline \ldots & 0 & 0 & 0 \\
\ldots & 1 & 0 & 0
\end{array}\right), \\
S_{3}=i\left(\begin{array}{cc|cc}
\ddots & \vdots & \vdots & \vdots \\
\ldots & 0 & 0 & 0 \\
\hline \ldots & 0 & 0 & 1 \\
\ldots & 0 & -1 & 0
\end{array}\right) ; \quad \phi=v(\vec{S} \cdot \widehat{r}) \phi(r)
\end{gathered}
$$

but this is a non-minimal monopole with mass

$$
M=\frac{8 \pi v}{g}
$$

twice that of the minimal ones. To summarize, there are $2 N$ degenerate minimally embedded monopoles. We note again that no irreducible representation of the gauge group $S O(2 N+1)$ has such a multiplicity, but this is precisely the multiplicity of the fundamental representation of the dual group, $\operatorname{USp}(2 N)$. 


\section{$\mathbf{5 . 2} S O(2 N+2) \rightarrow S O(2 N) \times U(1)$}

This breaking pattern may be achieved with the Higgs VEV:

$$
\phi=i\left(\begin{array}{ccc|cc}
0 & 0 & \ldots & 0 & 0 \\
0 & 0 & \ldots & 0 & 0 \\
\vdots & \vdots & \ddots & \vdots & \vdots \\
\hline 0 & 0 & \ldots & 0 & v \\
0 & 0 & \ldots & -v & 0
\end{array}\right)=i\left(\begin{array}{c|cc}
0 \cdot \mathbf{1}_{(2 N) \times(2 N)} & & \\
\hline & 0 & v \\
-v & 0
\end{array}\right) .
$$

We use minimally-embedded $S U(2)$ subgroups as in the previous case. The formulas for $S, \tilde{S}, \phi$ are the same. We find $2 N$ monopoles with mass:

$$
M=\frac{4 \pi v}{g}
$$

This is the right number of degenerate degrees of freedom for a monopole in the vector representation of the dual $S O(2 N)$ magnetic group.

\section{$\mathbf{5 . 3} U S p(2 N+2) \rightarrow U S p(2 N) \times U(1)$}

The generic $U S p(2 N)$ matrix in the fundamental representation has the following form:

$$
Z=\left(\begin{array}{cc}
\mathbb{A} & \mathbb{B} \\
\mathbb{B}^{*} & -\mathbb{A}^{t}
\end{array}\right)
$$

with $\mathbb{A}^{\dagger}=\mathbb{A}$ and $\mathbb{B}^{t}=\mathbb{B}$. The symmetry breaking field is:

$$
\phi=\left(\begin{array}{ccc|ccc}
0 & \ldots & 0 & 0 & \ldots & 0 \\
\vdots & \ddots & \vdots & \vdots & \ddots & \vdots \\
0 & \ldots & v & 0 & \ldots & 0 \\
\hline 0 & \ldots & 0 & 0 & \ldots & 0 \\
\vdots & \ddots & \vdots & \vdots & \ddots & \vdots \\
0 & \ldots & 0 & 0 & \ldots & -v
\end{array}\right)=\left(\begin{array}{cc|c}
0 \cdot \mathbf{1}_{N \times N} & & \\
& v & \\
\hline & 0 \cdot \mathbf{1}_{N \times N} & \\
& & \\
& & \\
& & \\
& &
\end{array}\right.
$$

There are three kinds of monopoles: 


\section{First type}

We use the $S U(2)$ subgroup with $\mathbb{B}=0$.

$$
\begin{aligned}
& S_{1}=\frac{1}{2}\left(\begin{array}{ccc|ccc}
\ddots & & & & \\
& 0 & 1 & & 0 & 0 \\
& 1 & 0 & & 0 & 0 \\
\hline & & \ddots & & \\
& 0 & 0 & & 0 & -1 \\
0 & 0 & & -1 & 0
\end{array}\right) ; \quad S_{2}=\frac{1}{2}\left(\begin{array}{ccc|ccc}
\ddots & & & & & \\
& 0 & -i & & & \\
& 0 & 0 \\
i & 0 & & 0 \\
& & & \ddots & & \\
& 0 & 0 & & 0 & i \\
& 0 & 0 & & -i & 0
\end{array}\right), \\
& S_{3}=\frac{1}{2}\left(\begin{array}{ccc|ccc}
\ddots & & & & & \\
& 1 & 0 & & 0 & 0 \\
& -1 & & 0 & 0 \\
\hline & & \ddots & & \\
0 & 0 & & -1 & 0 \\
0 & 0 & & 0 & 1
\end{array}\right)
\end{aligned}
$$

to construct the adjoint scalar profile

$$
\phi=\left(\begin{array}{ccc|ccc}
\ddots & & & & & \\
& v / 2 & 0 & & 0 & 0 \\
0 & v / 2 & & 0 & 0 \\
\hline & & \ddots & & \\
0 & 0 & & -v / 2 & 0 \\
& 0 & & 0 & -v / 2
\end{array}\right)-v(\vec{S} \cdot \widehat{r}) \phi(r) .
$$

There are $N$ monopoles of this kind, each with a mass of $M=\frac{4 \pi v}{g}$.

\section{Second type}

Alternately we may use the embedding

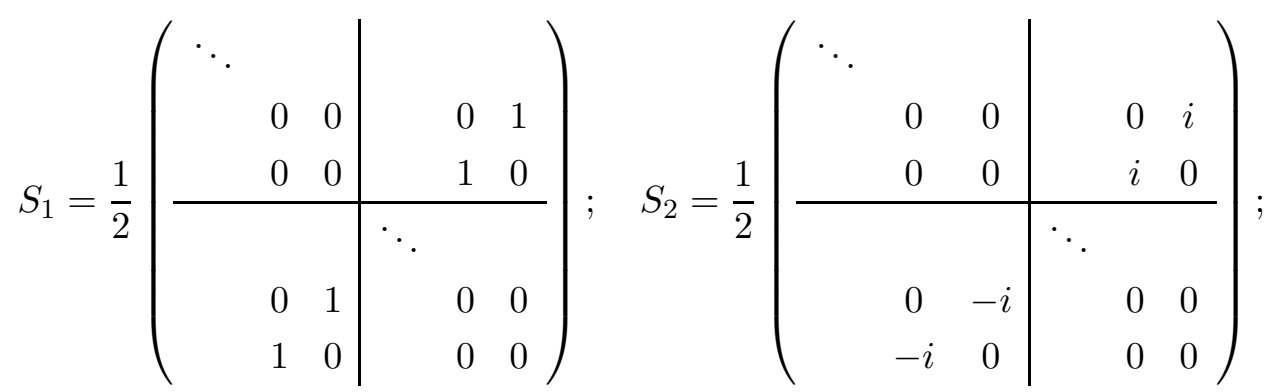




$$
S_{3}=\frac{1}{2}\left(\begin{array}{ccc|ccc}
\ddots & & & & & \\
& -1 & 0 & & 0 & 0 \\
0 & -1 & & 0 & 0 \\
\hline & & \ddots & & \\
0 & 0 & & 1 & 0 \\
0 & 0 & & 0 & 1
\end{array}\right)
$$

leading to an adjoint scalar profile

$$
\phi=\left(\begin{array}{ccc|ccc}
\ddots & & & & & \\
& -v / 2 & 0 & & 0 & 0 \\
0 & v / 2 & & 0 & 0 \\
\hline & & \ddots & & \\
0 & 0 & & v / 2 & 0 \\
0 & 0 & & 0 & -v / 2
\end{array}\right)-v(\vec{S} \cdot \widehat{r}) \phi(r) .
$$

There are again $N$ monopoles of this kind, each with $M=\frac{4 \pi v}{g}$.

\section{Third type}

The last monopole lies in the $S U(2)$ subgroup

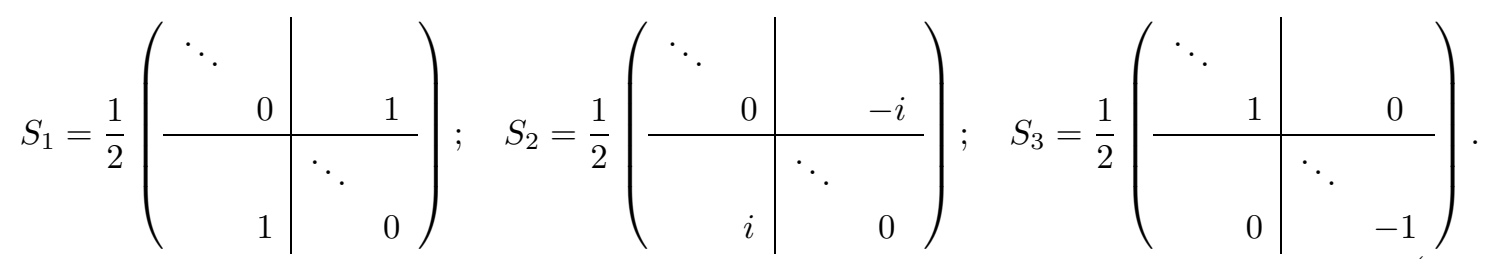

There is only one monopole of this type,

$$
\phi=2 v(\vec{S} \cdot \widehat{r}) \phi(r), \quad M=\frac{4 \pi v}{g} .
$$

To summarize, we have found $2 N+1$ degenerate monopoles states in the system $U S p(2 N+2) \rightarrow U S p(2 N) \times U(1)$. This is exactly the number of degrees of freedom for a monopole in the vector representation of the dual $S O(2 N+1)$ magnetic group. This case, like that of Subsec. 5.1 nicely illustrates the fact that soliton states of $U S p(2 N)$ gauge-invariant system appear with multiplicity appropriate for the dual group - $S O(2 N+1)$ in this case - and not with multiplicities of any irreducible representation of the original group $U S p(2 N)$. 


\section{$5.4 \quad S O(2 N) \rightarrow U(N)$}

This symmetry breaking pattern is characteristic of the adjoint scalar profile

$$
\phi=i\left(\begin{array}{cc|cc|cc}
0 & v & \ldots & \ldots & 0 & 0 \\
-v & 0 & \ldots & \ldots & 0 & 0 \\
\hline \vdots & \vdots & \ddots & & \vdots & \vdots \\
\vdots & \vdots & & \ddots & \vdots & \vdots \\
\hline 0 & 0 & \ldots & \ldots & 0 & v \\
0 & 0 & \ldots & \ldots & -v & 0
\end{array}\right) .
$$

Consider first of all the $S O(4) \rightarrow U(2)$ case. We use the basis $S_{i}, \tilde{S}_{i}$ in Eq. (5.2)-(5.5); these two $S U(2)$ subgroups are orthogonal and commute with each other. As the $\tilde{S}_{i}$ subgroup is actually unbroken we can build a monopole using the $S_{i}$ generators only:

$$
\phi=2 v(\vec{S} \cdot \widehat{r}) \phi(r),
$$

with a mass

$$
M=\frac{8 \pi v}{g} .
$$

In the general $S O(2 N) \rightarrow U(N)$ case, we find $\frac{N(N-1)}{2}$ degenerate monopoles, which can be interpreted as an antisymmetric 2-tensor representation of the (dual) $S U(N)$.

\section{$5.5 \quad S O(2 N+1) \rightarrow U(N)$}

This symmetry breaking pattern results from the adjoint Higgs VEV

$$
\phi=i\left(\begin{array}{cc|cc|cc|c}
0 & v & \ldots & \ldots & 0 & 0 & 0 \\
-v & 0 & \ldots & \ldots & 0 & 0 & 0 \\
\hline \vdots & \vdots & \ddots & & \vdots & \vdots & \vdots \\
\vdots & \vdots & & \ddots & \vdots & \vdots & \vdots \\
\hline 0 & 0 & \ldots & \ldots & 0 & v & 0 \\
0 & 0 & \ldots & \ldots & -v & 0 & 0 \\
\hline 0 & 0 & \ldots & \ldots & 0 & 0 & 0
\end{array}\right) .
$$

In this case we may again construct all $\frac{N(N-1)}{2}$ monopoles found above in the $S O(2 N) \rightarrow$ $U(N)$ system. But there are another $N$ monopoles corresponding to the following em- 
beddings

$$
\begin{gathered}
S_{1}=i\left(\begin{array}{c|cc|c}
\ddots & \vdots & \vdots & \vdots \\
\hline \ldots & 0 & 0 & 0 \\
\ldots & 0 & 0 & 1 \\
\hline \ldots & 0 & -1 & 0
\end{array}\right), \quad S_{2}=i\left(\begin{array}{c|cc|c}
\ddots & \vdots & \vdots & \vdots \\
\hline \ldots & 0 & 0 & -1 \\
\ldots & 0 & 0 & 0 \\
\hline \ldots & 1 & 0 & 0
\end{array}\right), \\
S_{3}=i\left(\begin{array}{c|cc|c}
\ddots & \vdots & \vdots & \vdots \\
\hline \ldots & 0 & 1 & 0 \\
\ldots & -1 & 0 & 0 \\
\hline \ldots & 0 & 0 & 0
\end{array}\right) .
\end{gathered}
$$

The scalar profile is then

$$
\phi=i\left(\begin{array}{cc|cc|cc|c}
0 & v & \ldots & \ldots & 0 & 0 & 0 \\
-v & 0 & \ldots & \ldots & 0 & 0 & 0 \\
\hline \vdots & \vdots & \ddots & & \vdots & \vdots & \vdots \\
\vdots & \vdots & & \ddots & \vdots & \vdots & \vdots \\
\hline 0 & 0 & \ldots & \ldots & 0 & 0 & 0 \\
0 & 0 & \ldots & \ldots & 0 & 0 & 0 \\
\hline 0 & 0 & \ldots & \ldots & 0 & 0 & 0
\end{array}\right)+v(\vec{S} \cdot \widehat{r}) \phi(r)
$$

and the mass :

$$
M=\frac{8 \pi v}{g}
$$

We then find in all $\frac{N(N+1)}{2}$ degenerate monopoles which transform in the symmetric 2-tensor representation of $S U(N)$.

\section{$\mathbf{5 . 6} U S p(2 N) \rightarrow U(N)$}

This gauge symmetry breaking pattern results from the Higgs VEV

$$
\phi=\left(\begin{array}{l|l}
v \cdot \mathbf{1}_{N \times N} & \\
\hline & -v \cdot \mathbf{1}_{N \times N}
\end{array}\right) .
$$

In this case we describe the two least massive species of monopole, one of which transforms in the fundamental representation of the dual $S U(n)$ and the other in the antisymmetric 2-tensor representation. 


\section{Fundamental Monopole}

We take the following $S U(2)$ subgroups:

$$
\begin{gathered}
S_{1}=\frac{1}{2}\left(\begin{array}{ccc|ccc}
0 & \ldots & 0 & 1 & \ldots & 0 \\
\vdots & \ddots & \vdots & \vdots & \ddots & \vdots \\
0 & \ldots & 0 & 0 & \ldots & 0 \\
\hline 1 & \ldots & 0 & 0 & \ldots & 0 \\
\vdots & \ddots & \vdots & \vdots & \ddots & \vdots \\
0 & \ldots & 0 & 0 & \ldots & 0
\end{array}\right) ; \quad S_{2}=\frac{1}{2}\left(\begin{array}{ccc|ccc}
0 & \ldots & 0 & -i & \ldots & 0 \\
\vdots & \ddots & \vdots & \vdots & \ddots & \vdots \\
0 & \ldots & 0 & 0 & \ldots & 0 \\
\hline i & \ldots & 0 & 0 & \ldots & 0 \\
\vdots & \ddots & \vdots & \vdots & \ddots & \vdots \\
0 & \ldots & 0 & 0 & \ldots & 0
\end{array}\right) ; \\
S_{3}=\frac{1}{2}\left(\begin{array}{ccc|ccc}
1 & \ldots & 0 & 0 & \ldots & 0 \\
\vdots & \ddots & \vdots & \vdots & \ddots & \vdots \\
0 & \ldots & 0 & 0 & \ldots & 0 \\
\hline 0 & \ldots & 0 & -1 & \ldots & 0 \\
\vdots & \ddots & \vdots & \vdots & \ddots & \vdots \\
0 & \ldots & 0 & 0 & \ldots & 0
\end{array}\right) .
\end{gathered}
$$

The resulting Higgs VEV is

$$
\phi=\left(\begin{array}{cccc|cccc}
0 & \ldots & \ldots & 0 & 0 & \ldots & \ldots & 0 \\
\vdots & v & & \vdots & \vdots & \ddots & & \vdots \\
\vdots & & \ddots & \vdots & \vdots & & \ddots & \vdots \\
0 & \ldots & \ldots & v & 0 & \ldots & \ldots & 0 \\
\hline 0 & \ldots & \ldots & 0 & 0 & \ldots & \ldots & 0 \\
\vdots & \ddots & & \vdots & \vdots & -v & & \vdots \\
\vdots & & \ddots & \vdots & \vdots & & \ddots & \vdots \\
0 & \ldots & \ldots & 0 & 0 & \ldots & \ldots & -v
\end{array}\right)+2 v(\vec{S} \cdot \widehat{r}) \phi(r) .
$$

These embeddings describe $N$ monopoles with the same mass $M=\frac{8 \pi v}{g}$. They transform in the fundamental representation of the dual $S U(N)$.

\section{Antisymmetric 2-Tensor Monopole}

If instead we consider the following $S U(2)$ subgroups:

$$
S_{1}=\left(\begin{array}{cc}
\mathbf{0} & \mathbf{A}_{1} \\
\mathbf{A}_{1}^{*} & \mathbf{0}
\end{array}\right), \quad S_{2}=\left(\begin{array}{cc}
\mathbf{0} & \mathbf{A}_{2} \\
\mathbf{A}_{2}^{*} & \mathbf{0}
\end{array}\right), \quad S_{3}=\left(\begin{array}{cc}
\mathbf{B}_{3} & \mathbf{0} \\
\mathbf{0} & -\mathbf{B}_{3}^{T}
\end{array}\right),
$$




$$
\begin{aligned}
& \mathbf{A}_{1}=\frac{1}{2}\left(\begin{array}{ccccc}
0 & \ldots & 1 & \ldots & 0 \\
\vdots & \ddots & & & \vdots \\
1 & & \ddots & & \vdots \\
\vdots & & & \ddots & \vdots \\
0 & \ldots & \ldots & \ldots & 0
\end{array}\right) ; \quad \mathbf{A}_{2}=\frac{1}{2}\left(\begin{array}{ccccc}
0 & \ldots & -i & \ldots & 0 \\
\vdots & \ddots & & & \vdots \\
-i & & \ddots & & \vdots \\
\vdots & & & \ddots & \vdots \\
0 & \ldots & \ldots & \ldots & 0
\end{array}\right) ; \\
& \mathbf{B}_{3}=\frac{1}{2}\left(\begin{array}{ccccc}
1 & \ldots & \ldots & \ldots & 0 \\
\vdots & \ddots & & & \vdots \\
\vdots & & 1 & & \vdots \\
\vdots & & & \ddots & \vdots \\
0 & \ldots & \ldots & \ldots & 0
\end{array}\right)
\end{aligned}
$$

then we may construct the solutions

$$
\phi=\left(\begin{array}{cc}
\mathbf{B}_{\phi} & \mathbf{0} \\
\mathbf{0} & -\mathbf{B}_{\phi}^{T}
\end{array}\right)+2 v(\vec{S} \cdot \widehat{r}) \phi(r)
$$

where

$$
\mathbf{B}_{\phi}=\left(\begin{array}{ccccc}
0 & \ldots & \ldots & \ldots & 0 \\
\vdots & v & & & \vdots \\
\vdots & & 0 & & \vdots \\
\vdots & & & v & \vdots \\
0 & \ldots & \ldots & \ldots & v
\end{array}\right)
$$

These solutions describe $\frac{N(N-1)}{2}$ degenerate monopoles that transform in the antisymmetric tensor representation of the dual $S U(N)$. Their mass is twice the mass of the fundamental monopole:

$$
M=\frac{16 \pi v}{g}
$$

\section{$5.7 \quad$ Others}

Other breaking patterns discussed in the previous section, $S U(N+M) \rightarrow S U(N) \times$ $S U(M) \times U(1) / \mathbb{Z}_{k}, S O(N+2 M) \rightarrow S O(N) \times U(M)$, and $U S p(2 N+2 M) \rightarrow$ $U S p(2 N) \times U(M)$, can be discussed basically by fixing the index of the latter gauge factor and carry through the same analysis as those in the similar breaking patterns presented in this section. 


\section{Summary and Discussion}

In this paper we have constructed magnetic monopoles arising in spontaneously broken gauge theories with several symmetry breaking patterns $G \rightarrow H$. Our work is based on the results of our predecessors [1]- 9], and should be regarded as a continuation of their efforts. Some overlap and repetition of the results discussed here with the earlier ones [1]-11] is inevitable. Nevertheless, we believe that the present work constitutes a qualitatively new contribution to this chain of developments.

Most importantly, the conditions under which these nonabelian monopoles survive quantum effects have been clarified, as is discussed in Sections 2 and 3 . In particular there is now strong evidence from $\mathcal{N}=2$ theories that these monopoles become light due to quantum effects and emerge as the dominant low-energy degrees of freedom in certain vacua; it was pointed out that the presence of an appropriate number of massless flavors in the underlying theory is crucial for this to happen. As illustrated in $S U(N)$ examples in Section 2 and discussed in a more general context in Section 3 , a consistent, quantum theory of nonabelian monopoles can be constructed by embed-

ding the system in $\mathcal{N}=2$ supersymmetric theories coupled to an appropriate set of hypermultiplets.

Although we considered in this paper unconfined monopoles mainly, a rather surprising hint about the nature of nonabelian monopoles comes from considering the systems in which the "unbroken" group $H$ is further broken by some other VEVs at a much smaller mass scale. The nonabelian monopoles whose gauge bundles are described by a transition function in $\pi_{1}(H)$ are confined by the nonabelian vortices whose squark profile wraps the same class in $\pi_{1}(H)$, in the Higgs phase of the $H$ theory. In Ref. [21] it is demonstrated that the vortices of the low-energy system are described by a continuous family of solutions transforming under an irreducible representation of the dual group $\tilde{H}$. The monopole-vortex flux matching argument presented in [26] and proven explicitly for the case

$$
S U(N+1) \stackrel{v_{1}}{\longrightarrow} \frac{S U(N) \times U(1)}{\mathbb{Z}_{N}} \stackrel{v_{2}}{\longrightarrow} 0, \quad v_{1} \gg v_{2},
$$

implies that the monopoles corresponding to all classes in $\pi_{1}(H)$ are indeed confined. The existence of a continuous degenerate family of the vortices $\pi_{1}(H)$ implies a corresponding, continuous family of their sources: the monopoles. Most interestingly, the existence of the quantum mechanical vortex zero modes also requires nontrivial flavors: the dual group itself involves the original flavor group [21, 26]. 
Three complementary approaches: (i) topological arguments in which monopole field configurations are classified by homotopy groups (Section 4); (ii) general formulae à la GNO-Weinberg, making use of the root system structures of the groups (Appendix A), and (iii) the explicit construction of BPS solutions (Section 5), have been used in the present paper. Each of these approaches has some advantage over the others. The (topological) stability of the monopoles as well as the $U(1)$ charge as compared with the minimum Dirac quantum are best explained in the first approach. On the other hand, the multiplet structure of the minimal monopoles, and the reason why they form an irreducible multiplet of the dual group $\tilde{H}$, is best understood in the second approach and easily seen in the third. Finally the explicit construction (iii) provides a check of the whole program and furthermore is the simplest way (except for MQCD, which yields the same answers) to compute the monopole masses and representations. The calculation of the $U(1)$ magnetic charge is explained in Appendix A

The results for the quantum numbers of the minimal nonabelian monopoles in various cases are summarized in Table 3. Note that in some cases the minimal nonabelian monopoles do not belong to the fundamental representation of the unbroken (dual) group.

The pattern of the monopole representations appearing in various cases found here can be understood based on the Montonen-Olive duality of $\mathcal{N}=4$ models. Because of the self-duality, the representation of the monopoles under the dual group must be the same as that of the massive vector bosons with respect to the original, electric group. For instance, when $S O(2 N) \rightarrow U(N)$, the massive vector bosons are in the anti-symmetric tensor of $U(N)$, and so are indeed monopoles. When $U S p(2 N+2) \rightarrow$ $U S p(2 N) \times U(1)$, the dual theory breaks from $S O(2 N+3) \rightarrow S O(2 N+1) \times S O(2)$, and the massive vector bosons are in the vector representation. Indeed, we find monopoles in the vector representation of $S O(2 N+1)$.

A more non-trivial example is the breaking $S O(2 N+1) \rightarrow U(N)$, where the electric theory has monopoles in the rank-two symmetric tensor representation of $U(N)$, while the magnetic $U S p(2 N) \rightarrow U(N)$ theory has massive vectors in the same representation. The electric $S O(2 N+1)$ theory has massive vector bosons in the fundamental and anti-fundamental representations of $U(N)$, as well as in rank-two anti-symmetric tensor representations that are twice as heavy. On the other hand, the magnetic $U S p(2 N)$ theory has monopoles in the fundamental, anti-fundamental 


\begin{tabular}{|c|c|c|c|c|}
\hline $\mathbf{G}$ & $\mathbf{H}$ & Dual Group & Irrep & $U(1)$ \\
\hline$S U(N+1)$ & $S U(N) \times U(1) / \mathbb{Z}_{N}$ & $S U(N) \times U(1)$ & $\underline{N}$ & $1 / N$ \\
\hline$* S U(N+1)$ & $S U(r) \times U(1)^{N-r} / \mathbb{Z}_{r}$ & $S U(r) \times U(1)^{N-r+1}$ & $\underline{r}$ & $1 / r$ \\
\hline$U S p(2 N+2)$ & $U S p(2 N) \times U(1)$ & $S O(2 N+1) \times U(1)$ & $\underline{2 N+1}$ & 1 \\
\hline$* U S p(2 N+2)$ & $S U(r) \times U(1)^{N-r} / \mathbb{Z}_{r}$ & $S U(r) \times U(1)^{N-r+1}$ & $\underline{r}$ & $1 / r$ \\
\hline$U S p(2 N)$ & $S U(N) \times U(1) / \mathbb{Z}_{N}$ & $S U(N) \times U(1)$ & $\underline{N}$ & $1 / N$ \\
\hline$S O(2 N+3)$ & $S O(2 N+1) \times U(1)$ & $U S p(2 N) \times U(1)$ & $\underline{2 N}$ & 1 \\
\hline$S O(2 N+2)$ & $S O(2 N) \times U(1)$ & $S O(2 N) \times U(1)$ & $\underline{2 N}$ & 1 \\
\hline$S O(2 N)$ & $S U(N) \times U(1) / \mathbb{Z}_{N}$ & $S U(N) \times U(1)$ & $\underline{N(N-1) / 2}$ & $2 / N$ \\
\hline$* S O(2 N)$ & $S U(r) \times U(1)^{N-r+1} / \mathbb{Z}_{r}$ & $S U(r) \times U(1)^{N-r+1}$ & $\underline{r}$ & $1 / r$ \\
\hline$S O(2 N+1)$ & $S U(N) \times U(1) / \mathbb{Z}_{N}$ & $S U(N) \times U(1)$ & $\underline{N(N+1) / 2}$ & $2 / N$ \\
\hline$* S O(2 N+1)$ & $S U(r) \times U(1)^{N-r+1} / \mathbb{Z}_{r}$ & $S U(r) \times U(1)^{N-r+1}$ & $\underline{r}$ & $1 / r$ \\
\hline$S U(N+M)$ & $S U(N) \times S U(M) \times U(1) / \mathbb{Z}_{k}$ & $S U(N) \times S U(M) \times U(1)$ & $(\underline{N}, \underline{\bar{M}})$ & $1 / k$ \\
\hline$S O(2 N+2 M)$ & $S O(2 N) \times U(M)$ & $S O(2 N) \times U(M)$ & $(\underline{2 N}, \underline{M})$ & $1 / M$ \\
\hline$S O(2 N+2 M+1)$ & $S O(2 N+1) \times U(M)$ & $U S p(2 N) \times U(M)$ & $(\underline{2 N}, \underline{M})$ & $1 / M$ \\
\hline$U S p(2 N+2 M)$ & $U S p(2 N) \times U(M)$ & $S O(2 N+1) \times U(M)$ & $(2 N+1, M)$ & $1 / M$ \\
\hline
\end{tabular}

Table 3: Stable nonabelian magnetic monopoles of minimum mass arising from the breaking $G \rightarrow H$ and their charges. The $U(1)$ magnetic charge is given in the unit of the minimum Dirac quantum, $1 / 2 e_{0}$, where $e_{0}$ is the minimum electric $U(1)$ charge in the system. $k$ in the $S U(N+M)$ case is the least common multiple of $N$ and $M$. The cases with nonmaximal nonabelian factors $\left(^{*}\right), r<N$, are qualitatively different from the case with $r=N$ in that monopoles in the fundamental as well as in the second-rank tensor representation of $S U(r)$ appear: only the monopoles in the fundamental representation of $S U(r)$ are indicated. Note that these do not exist for $r=N$ in the case of $G=S O(N)$, as most easily seen from the explicit construction of Sec. 5.4 and Sec. 5.5.

representation with the minimum charge, as well as in rank-two anti-symmetric tensor with twice as large charge and hence twice as large mass (they are not in Table 3 because they can decay into monopoles in the fundamental representation; see Section 5.6). This agreement is also manifest in MQCD, where the representations are determined by the same orientifold projections, employing O3-planes for $\mathcal{N}=4$ and O4-planes for $\mathcal{N}=2$.

Finally, the consistency with Olive-Montonen duality can be checked also in the cases with two nonabelian factors in $H$. For instance, in the system $S U(N+M) \rightarrow$ $S U(N) \times S U(M) \times U(1) / \mathbb{Z}_{k}$ both the massive gauge bosons of the dual theory and the monopoles of the electric theory are in the $(\underline{N}, \underline{\bar{M}})$ representation. Analogously for the 
case, $S O(2 N+2 M) \rightarrow S O(2 N) \times U(M)$. In the case $S O(2 N+2 M+1) \rightarrow S O(2 N+$ 1) $\times U(M)$, the dual theory breaks as $U S p(2 N+2 M) \rightarrow U S p(2 N) \times U(M)$, generating the massive gauge bosons in the $(\underline{2 N}, \underline{M})$ representation, consistently with our description in terms of the monopoles in the electric theory. Finally, the monopoles in the $(\underline{2 N+1}, \underline{M})$ found in the system $U S p(2 N+2 M) \rightarrow U S p(2 N) \times U(M)$, has an equivalent, dual description in terms of massive gauge bosons of the dual theory $S O(2 N+2 M+1) \rightarrow S O(2 N+1) \times U(M)$.

Another, independent consistency check of our general results can be made by using the (Lie algebra) isomorphism between the groups $U S p(4)$ and $S O(5)$. Consider the $U S p(4)$ theory with two different symmetry breaking patterns

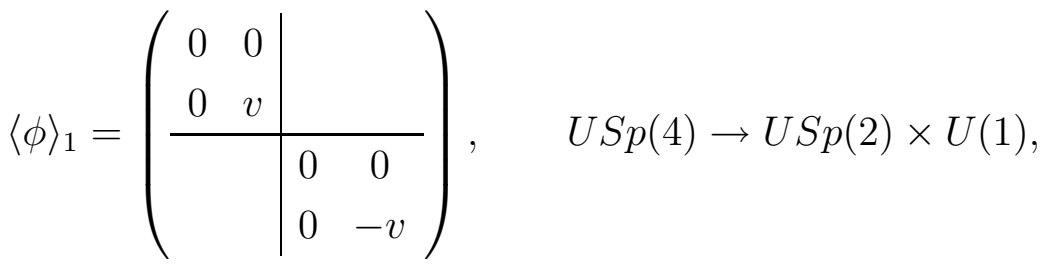

$$
\begin{aligned}
& \langle\phi\rangle_{2}=\left(\begin{array}{cc|cc}
v & 0 & & \\
0 & v & & \\
\hline & -v & 0 \\
& 0 & -v
\end{array}\right), \quad U S p(4) \rightarrow U(2) .
\end{aligned}
$$

In the first system, we find (see Table 3, Section 5) that the minimal monopoles are in the vector ( $\underline{3})$ of the $S O(3)$ group, which is dual to $U S p(2)$, while in the second system the minimal monopoles are in the fundamental representation (2) of the dual $S U(2)$ group, but there are also monopoles in the antisymmetric representation ( 1 in this case) with twice the minimal mass.

In the $S O(5)$ theory, again one has two inequivalent ways to break the gauge symmetry,

$$
\langle\phi\rangle_{1}=\left(\begin{array}{cc|cc|c}
0 & v & & & \\
-v & 0 & & & \\
\hline & & 0 & v & \\
& -v & 0 & \\
\hline & & & & 0
\end{array}\right), \quad S O(5) \rightarrow U(2)
$$




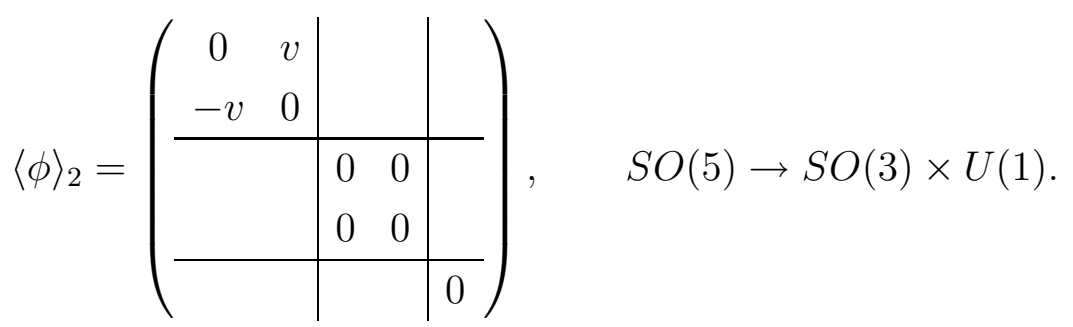

According to our general results, the monopoles are in the symmetric second rank tensor representation ( $\underline{3})$ of $S U(2)$ in the first system while in the second case they are fundamentals $(\underline{2})$ of the dual group $U S p(2) \sim S U(2)$ of $S O(3)$. In this last case there is also a non-minimal monopole of mass twice the minimal value.

The monopole spectra in the two theories thus agree completely, as expected. Actually, the correspondence can be shown to be exact: the first (second) pattern of the symmetry breaking in the $U S p(4)$ theory corresponds to the second (first) type of the adjoint $\mathrm{VEV}$ in the $S O(4)$ theory.

The whole discussion can actually be understood very easily from the general formula discussed in Appendix A, as the root vector system of the $U S p(4)$ group corresponds simply to a 45 degree rotation of that of $S O(5)$ in the bases used above as seen in Figure 2. The mass spectrum may be read off of this figure as well.

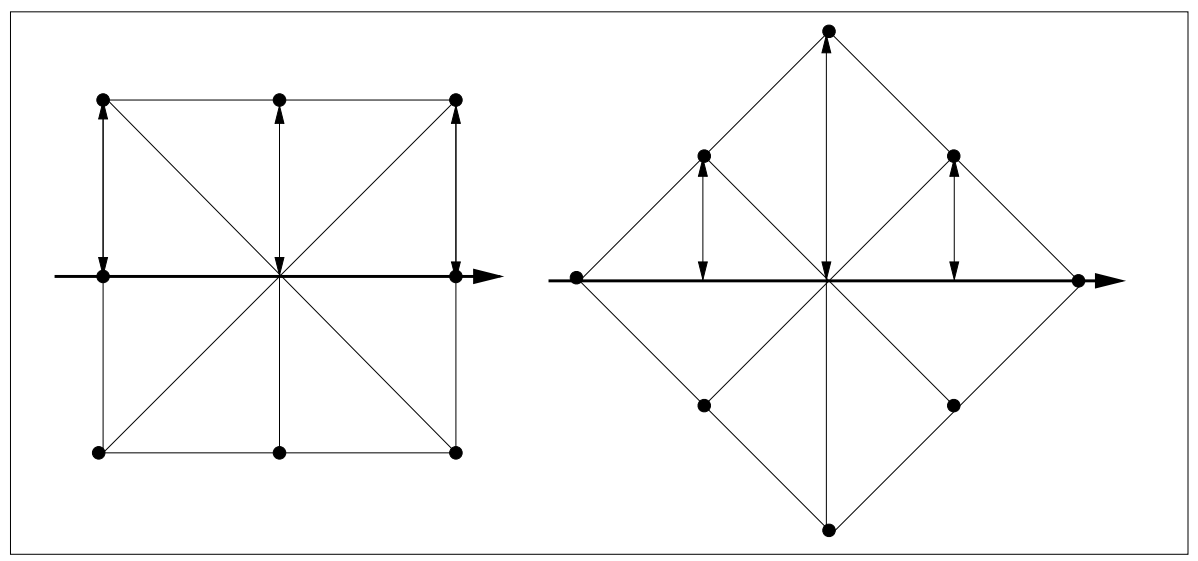

Figure 2: Root vectors of $S O(5)$ (left) and $U S p(4)$ (right). The root vectors in the horizontal directions represent the unbroken $S U(2)$ group in the case of the first breaking pattern (Eq.(6.2) ) for $S O(5)$ and the second breaking pattern (Eq.(6.5)) for $U S p(4)$. The masses of the monopoles are proportional to the heights of the (broken) root vectors.

An analogous check, using the isomorphism between $S O(6)$ and $S U(4)$ which are spontaneously broken respectively to $S O(4) \times U(1)$ and $S U(2) \times S U(2) \times U(1)$, also 
yields consistent results: the monopoles are in the $\underline{4}=(\underline{2}, \underline{2})$ of $S O(4) \sim S U(2) \times$ $S U(2)$. Another possibility is to consider the breaking patterns $S O(6) \rightarrow S U(3) \times$ $U(1)$ and $S U(4) \rightarrow S U(3) \times U(1)$. Again, we learn from Table 3 that the minimal monopoles belong to a triplet (or an antitriplet) of the unbroken $S U(3)$ in both theories, consistently.

\section{Acknowledgement}

We thank N. Dorey, A. Hanany, H. Hansson, K. Higashijima, A. Ritz and D. Tong for useful discussions and comments. The work of HM was supported by the Institute

for Advanced Study, funds for Natural Sciences, as well as in part by the DOE under contracts DE-AC03-76SF00098 and in part by NSF grant PHY-0098840. We thank also Sander Bais for useful communications.

\section{References}

[1] E. Lubkin, Ann. Phys. 23 (1963) 233.

[2] T. T. Wu and C. N. Yang, Phys. Rev. D12 (1975) 3845.

[3] C. Montonen and D. Olive, Phys. Lett. 72 B (1977) 117.

[4] P. Goddard, J. Nuyts and D. Olive, Nucl. Phys. B125 (1977) 1.

[5] F.A. Bais, Phys. Rev. D18 (1978) 1206; B.J. Schroers and F.A. Bais, Nucl. Phys. B512 (1998) 250, hep-th/9708004 Nucl. Phys. B535 (1998) 197, hep-th/9805163.

[6] E. J. Weinberg, Nucl. Phys. B167 (1980) 500; Nucl. Phys. B203 (1982) 445.

[7] S. Coleman, "The Magnetic Monopole Fifty Years Later," Lectures given at Int. Sch. of Subnuclear Phys., Erice, Italy (1981).

[8] Chan Hong-Mo and Tsou Sheung Tsun, Nucl. Phys. B189 (1981) 364.

[9] K. Lee, E. J. Weinberg and P. Yi, Phys. Rev. D 54 (1996) 6351, hep-th/9605229. 
[10] A. Abouelsaood, Nucl. Phys. B226 (1983) 309; P. Nelson and A. Manohar, Phys. Rev. Lett. 50 (1983) 943; A. Balachandran, G. Marmo, M. Mukunda, J. Nilsson, E. Sudarshan and F. Zaccaria, Phys. Rev. Lett. 50 (1983) 1553; P. Nelson and S. Coleman, Nucl. Phys. B227 (1984) 1.

[11] C. J. Houghton, P. M. Sutcliffe, J.Math.Phys.38 (1997) 5576, hep-th/9708006.

[12] N. Seiberg, Nucl.Phys.B435 (1995) 129, hep-th/9411149 D. Kutasov, A. Schwimmer and N. Seiberg, Nucl.Phys.B459 (1996) 455, hep-th/9510222.

[13] N. Seiberg and E. Witten, Nucl. Phys. B426 (1994) 19; Erratum ibid. Nucl.Phys. B430 (1994) 485, hep-th/9407087.

[14] N. Seiberg and E. Witten, Nucl. Phys. B431 (1994) 484, hep-th/9408099.

[15] P. C. Argyres and A. F. Faraggi, Phys. Rev. Lett 74 (1995) 3931, hep-th/9411047; A. Klemm, W. Lerche, S. Theisen and S. Yankielowicz, Phys. Lett. B344 (1995) 169, hep-th/9411048 Int. J. Mod. Phys. A11 (1996) 1929, hep-th/9505150 A. Hanany and Y. Oz, Nucl. Phys. B452 (1995) 283, hep-th/9505075; P. C. Argyres, M. R. Plesser and A. D. Shapere, Phys. Rev. Lett. 75 (1995) 1699, hep-th/9505100 P. C. Argyres and A. D. Shapere, Nucl. Phys. B461 (1996) 437, hep-th/9509175, A. Hanany, Nucl.Phys. B466 (1996) 85, hep-th/9509176.

[16] P. C. Argyres, M. R. Plesser and N. Seiberg, Nucl. Phys. B471 (1996) 159, hep-th/9603042 P.C. Argyres, M.R. Plesser, and A.D. Shapere, Nucl. Phys. B483 (1997) 172, hep-th/9608129.

[17] G. Carlino, K. Konishi and H. Murayama, JHEP 0002 (2000) 004, hep-th/0001036 Nucl. Phys. B590 (2000) 37, hep-th/0005076.

[18] G. Carlino, K. Konishi, Prem Kumar and H. Murayama, Nucl. Phys. B608 (2001) 51, hep-th/0104064.

[19] S. Bolognesi and K. Konishi, Nucl. Phys. B645 (2002) 337, hep-th/0207161.

[20] R. Auzzi, R. Grena and K. Konishi, Nucl. Phys. B653 (2003) 204, hep-th/0211282; R. Auzzi and R. Grena, hep-th/0402213.

[21] R. Auzzi, S. Bolognesi, J. Evslin, K. Konishi and A. Yung, Nucl. Phys. B673 (2003) 187, hep-th/0307287. 
[22] A. Hanany, D. Tong, JHEP 0307 (2003) 037, hep-th/0306150, D. Tong, Phys. Rev. D69 (2004) 065003, hep-th/0307302.

[23] M. A. C. Kneipp, Phys. Rev D68 (2003) 45009, hep-th/0211146 Phys. Rev. D69 (2004), hep-th/0308086.

[24] A. Hanany and D. Tong, JHEP 0404 (2004) 066, hep-th/0403158.

[25] M. Shifman and A. Yung, hep-th/0403149.

[26] R. Auzzi, S. Bolognesi, J. Evslin and K. Konishi, to appear in Nucl. Phys. B, hep-th/0312233.

[27] M.R. Douglas and S.H. Shenker, Nucl. Phys. B447 (1995) 271, hep-th/9503163.

[28] E. B. Bogomol'nyi, Sov. J. Nucl. Phys. 24 (1976) 449, M. K. Prasad and C. M. Sommerfield, Phys. Rev. Lett. 35 (1075) 760; S. Coleman, S. Parke, A. Neveu and C. M. Sommerfield, Phys. Rev. D15 (1977) 544.

[29] P.A.M. Dirac, Proc. Roy. Soc. A133 (1955) 60.

[30] G. 't Hooft, Nucl. Phys. B79 (1974) 276, A. M. Polyakov, JETP Lett. 20 (1974) 194.

[31] T. Eguchi, K. Hori, K. Ito and S.-K. Yang, Nucl. Phys. B471 (1996) 430, hep-th/9603002.

[32] P. C. Argyres and M. R. Douglas, Nucl. Phys. B448 (1995) 93, hep-th/9505062 P. C. Argyres, M. R. Plesser, N. Seiberg and E. Witten, Nucl. Phys. 461 (1996) 71, hep-th/9511154.

[33] R. Jackiw and C. Rebbi, Phys. Rev. D13 (1976) 3398.

[34] C. W. Bernard, N. H. Christ, A. H. Guth and E. J. Weinberg, Phys. Rev. D16 (1977) 2967. 


\section{Appendix A General Formulae}

In this appendix we review some general formulae [6, 4]. Nonabelian monopoles appear in a system with the gauge symmetry breaking

$$
G \stackrel{\langle\phi\rangle \neq 0}{\longrightarrow} H
$$

with a nontrivial $\pi_{2}(G / H)$ and $H$ nonabelian.

The normalization of the generators can be chosen [4] so that the metric of the root vector space is ${ }^{10}$

$$
g_{i j}=\sum_{\text {roots }} \alpha_{i} \alpha_{j}=\delta_{i j}
$$

The Higgs field vacuum expectation value (VEV) is taken to be of the form

$$
\phi_{0}=\mathbf{h} \cdot \mathbf{H}
$$

where $\mathbf{h}=\left(h_{1}, \ldots, h_{\operatorname{rank}(G)}\right)$ is a constant vector representing the VEV. The root vectors orthogonal to $\mathbf{h}$ belong to the unbroken subgroup $H$.

The monopole solutions are constructed from various $S U(2)$ subgroups of $G$ that do not commute with $H$,

$$
S_{1}=\frac{1}{\sqrt{2 \alpha^{2}}}\left(E_{\alpha}+E_{-\alpha}\right) ; \quad S_{2}=-\frac{i}{\sqrt{2 \alpha^{2}}}\left(E_{\alpha}-E_{-\alpha}\right) ; \quad S_{3}=\alpha^{*} \cdot \mathbf{H}
$$

where $\alpha$ is a root vector associated with a pair of broken generators $E_{ \pm \alpha} . \alpha^{*}$ is a dual root vector defined by

$$
\alpha^{*} \equiv \frac{\alpha}{\alpha \cdot \alpha} .
$$

The symmetry breaking (A.1) induces the Higgs mechanism in such an $S U(2)$ subgroup, $S U(2) \rightarrow U(1)$. By embedding the known 't Hooft-Polyakov monopole [30, 28, lying in this subgroup and adding a constant term to $\phi$ so that it behaves correctly asymptotically, one easily constructs a solution of the equation of motion [6, 19]:

$$
A_{i}(\mathbf{r})=A_{i}^{a}(\mathbf{r}, \mathbf{h} \cdot \alpha) S_{a} ; \quad \phi(\mathbf{r})=\chi^{a}(\mathbf{r}, \mathbf{h} \cdot \alpha) S_{a}+\left[\mathbf{h}-(\mathbf{h} \cdot \alpha) \alpha^{*}\right] \cdot \mathbf{H},
$$

${ }^{10}$ In the Cartan basis the Lie algebra of the group $G$ takes the form

$$
\begin{gathered}
{\left[H_{i}, H_{k}\right]=0, \quad(i, k=1,2, \ldots, r) ; \quad\left[H_{i}, E_{\alpha}\right]=\alpha_{i} E_{\alpha} ; \quad\left[E_{\alpha}, E_{-\alpha}\right]=\alpha^{i} H_{i} ;} \\
{\left[E_{\alpha}, E_{\beta}\right]=N_{\alpha \beta} E_{\alpha+\beta} \quad(\alpha+\beta \neq 0) .}
\end{gathered}
$$

$\alpha_{i}=\left(\alpha_{1}, \alpha_{2}, \ldots\right)$ are the root vectors. 
where

$$
A_{i}^{a}(\mathbf{r})=\epsilon_{a i j} \frac{r^{j}}{r^{2}} A(r) ; \quad \chi^{a}(\mathbf{r})=\frac{r^{a}}{r} \chi(r), \quad \chi(\infty)=\mathbf{h} \cdot \alpha
$$

is the standard 't Hooft-Polyakov-BPS solution. Note that $\phi(\mathbf{r}=(0,0, \infty))=\phi_{0}$.

The mass of a BPS monopole is then given by

$$
M=\int d \mathbf{S} \cdot \operatorname{Tr} \phi \mathbf{B}, \quad \mathbf{B}=\frac{r_{i}(\mathbf{S} \cdot \mathbf{r})}{r^{4}} .
$$

This can be computed by going to the gauge in which

$$
\mathbf{B}=\frac{\mathbf{r} S_{3}}{r^{3}}=\frac{\mathbf{r}}{r^{3}} \alpha^{*} \cdot \mathbf{H}
$$

to be

$$
M=\frac{4 \pi h_{i} \alpha_{j}^{*}}{g} \operatorname{Tr} H_{i} H_{j}
$$

For instance, the mass of the minimal monopole of $S U(N+1) \rightarrow S U(N) \times U(1)$ can be found easily by using Eqs.(B.4 $-($ B.10)

$$
M=\frac{2 \pi v(N+1)}{g} .
$$

For the cases $S O(N+2) \rightarrow S O(N) \times U(1)$ and $U S p(2 N+2) \rightarrow U S p(2 N) \times U(1)$, where $\operatorname{Tr} H_{i} H_{j}=C \delta_{i j}$, one finds

$$
M=\frac{4 \pi C \mathbf{h} \cdot \alpha^{*}}{g}=\frac{4 \pi v}{g},
$$

while for $S O(2 N) \rightarrow S U(N) \times U(1), S O(2 N+1) \rightarrow S U(N) \times U(1)$, and $U S p(2 N) \rightarrow$ $S U(N) \times U(1)$, the mass is

$$
M=\frac{8 \pi C \mathbf{h} \cdot \alpha^{*}}{g}=\frac{8 \pi v}{g} .
$$

In order to get the $U(1)$ magnetic charge ${ }^{11}$ (the last column of Table 31), we first divide by an appropriate normalization factor in the mass formula Eq.(A.10)

$$
F_{m}=\int d \mathbf{S} \cdot \frac{\operatorname{Tr} \phi \mathbf{B}}{N_{\phi}}=\int d \mathbf{S} \cdot \mathbf{B}^{(0)}, \quad \mathbf{B}=\frac{r_{i}(\mathbf{S} \cdot \mathbf{r})}{r^{4}}
$$

as was done in Eq.(2.14). The result, which is equal to $4 \pi g_{m}$ by definition, gives the magnetic charge. The latter must then be expressed as a function of the minimum

\footnotetext{
${ }^{11}$ In this calculation it is necessary to use the generators normalized as $\operatorname{Tr} T^{(a)} T^{(b)}=\frac{1}{2} \delta_{a b}$, such that $\mathbf{B}=\mathbf{B}^{(0)} T^{(0)}+\ldots$
} 
$U(1)$ electric charge present in the given theory, which can be easily found from the normalized (such that $\operatorname{Tr} T^{(a)} T^{(a)}=\frac{1}{2}$ ) form of the relevant $U(1)$ generator.

For example, in the case of the symmetry breaking, $S O(2 N) \rightarrow U(N)$, the adjoint VEV is of the form, $\phi=\sqrt{4 N} v T^{(0)}$, where $T^{(0)}$ is a $2 N \times 2 N$ block-diagonal matrix with $N$ nonzero submatrices $\frac{i}{\sqrt{4 N}}\left(\begin{array}{cc}0 & 1 \\ -1 & 0\end{array}\right)$. Dividing the mass (A.15) by $\sqrt{N} v$ and identifying the flux with $4 \pi g_{m}$ one gets $g_{m}=\frac{2}{\sqrt{N} g}$. Finally, in terms of the minimum electric charge of the theory $e_{0}=\frac{g}{\sqrt{4 N}}$ ( which follows from the normalized form of $T^{(0)}$ above) one finds

$$
g_{m}=\frac{2}{\sqrt{N} g}=\frac{2}{N} \cdot \frac{1}{2 e_{0}} .
$$

The calculation is similar in other cases.

The asymptotic gauge field can be written as

$$
F_{i j}=\epsilon_{i j k} B_{k}=\epsilon_{i j k} \frac{r_{k}}{r^{3}}(\beta \cdot \mathbf{H}), \quad \beta=\alpha^{*}
$$

in an appropriate gauge (Eq.(A.10) ). The Goddard-Nuyts-Olive quantization condition [4]

$$
2 \beta \cdot \alpha \in \mathbf{Z}
$$

then reduces to the well-known theorem that for two root vectors $\alpha_{1}, \alpha_{2}$ of any group,

$$
\frac{2\left(\alpha_{1} \cdot \alpha_{2}\right)}{\left(\alpha_{1} \cdot \alpha_{1}\right)}
$$

is an integer.

\section{Appendix B Root vectors and weight vectors}

\section{B.1 $A_{N}=S U(N+1)$}

It is sometimes convenient to have the root vectors and weight vectors of the Lie algebra $S U(N+1)$ as vectors in an $(N+1)$-dimensional space rather than an $N$ dimensional one. The root vectors are then simply

$$
(\cdots, \pm 1, \cdots, \mp 1, \cdots) .
$$

$(\cdots$ stand for zero elements) which all lie on the plane

$$
x_{1}+x_{2}+\ldots+x_{N+1}=0,
$$


while the weight vectors are projections in this plane of the orthogonal vectors

$$
\vec{\mu}=(\cdots, \pm 1, \cdots)
$$

where the dots represent zero elements.

In order to use the general formulas of Weinberg and Goddard-Olive-Nuyts we normalize these vectors so that the diagonal (Cartan) generators may be written

$$
\mathbf{H}_{\mathbf{i}}=\operatorname{diag}\left(w_{1}^{i}, w_{2}^{i} \ldots, w_{N}^{i}, w_{N+1}^{i}\right), \quad i=1,2 \ldots N
$$

where $w_{k}$ represents the $k$-th weight vector of the fundamental representation of $S U(N+1)$, satisfying

$\mathbf{w}_{k} \cdot \mathbf{w}_{l}=-\frac{1}{2(N+1)^{2}} ; \quad(k \neq l) ; \quad \mathbf{w}_{k} \cdot \mathbf{w}_{k}=\frac{N}{2(N+1)^{2}}, \quad k, l=1,2, \ldots, N+1 ;$

and $\sum_{k=1}^{N+1} \mathbf{w}_{k}=0$. They are vectors lying in an $N$-dimensional space $(\mathbb{B . 2})$ : in the coordinates of the $N+1$-dimensional space,

$$
\mathbf{w}_{i}=\frac{1}{\sqrt{2(N+1)^{3}}}(-1, \ldots,-1, N,-1,-1, \ldots) .
$$

The root vectors are simply

$$
\alpha=\mathbf{w}_{i}-\mathbf{w}_{j}=\frac{1}{\sqrt{2(N+1)}}(\cdots, \pm 1, \cdots, \mp 1, \cdots)
$$

with the norm

$$
\alpha \cdot \alpha=\frac{1}{N+1}
$$

Note that for $i \neq j$

$$
\operatorname{Tr}\left(H_{i} H_{j}\right)=w_{1}^{i} w_{1}^{j}+\ldots+w_{N+1}^{i} w_{N+1}^{j}=\frac{-2 N+N-1}{2(N+1)^{3}}=-\frac{1}{2(N+1)^{2}},
$$

while

$$
\operatorname{Tr}\left(H_{i} H_{i}\right)=\frac{N^{2}+N}{2(N+1)^{3}}=\frac{N}{2(N+1)^{2}} .
$$

The adjoint VEV causing the symmetry breaking $S U(N+1) \rightarrow S U(N) \times U(1)$ is of the form,

$$
\phi=\mathbf{h} \cdot \mathbf{H}, \quad \mathbf{h}=v \sqrt{2(N+1)^{3}}(0,0, \ldots, 1) .
$$




\section{B.2 $\quad B_{N}=S O(2 N+1)$}

The $N$ generators in the Cartan subalgebra of the Lie algebra $S O(2 N+1)$ can be taken to be

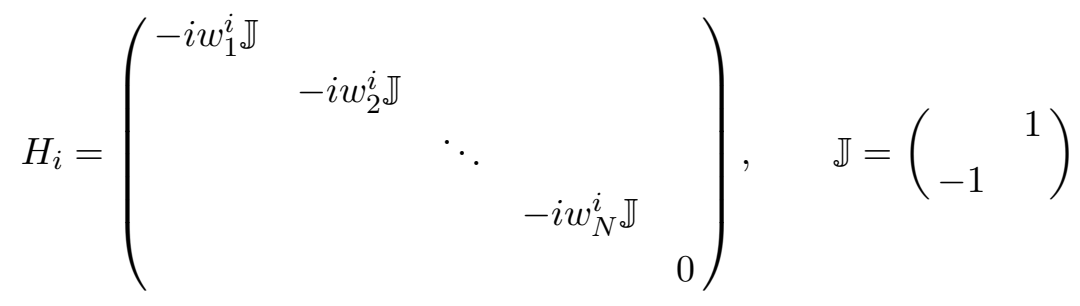

where $\mathbf{w}_{k}(k=1,2, \ldots, N)$ are the weight vectors of the fundamental representation, which are vectors in an $N$-dimensional Euclidean space

$$
\mathbf{w}_{k} \cdot \mathbf{w}_{l}=0 ; \quad k \neq l ; \quad \mathbf{w}_{k} \cdot \mathbf{w}_{k}=\frac{1}{2(2 N-1)}:
$$

they form a complete set of orthogonal vectors. The root vectors of $S O(2 N+1)$ group are $\alpha=\left\{ \pm \mathbf{w}_{i}, \pm \mathbf{w}_{i} \pm \mathbf{w}_{j}\right\}$; their duals are:

$$
\alpha^{*}= \pm 2(2 N-1) \mathbf{w}_{i}, \quad(2 N-1)\left[ \pm \mathbf{w}_{i} \pm \mathbf{w}_{j}\right] .
$$

The diagonal generators satisfy

$$
\operatorname{Tr} H_{i} H_{j}=\frac{1}{2 N-1} \delta_{i j} .
$$

In the system with symmetry breaking $S O(2 N+1) \rightarrow S O(2 N-1) \times U(1)$ the adjoint scalar $\mathrm{VEV}$ is

$$
\phi=\mathbf{h} \cdot \mathbf{H}, \quad \mathbf{h}=i v \sqrt{2(2 N-1)}(0,0, \ldots, 1)
$$

\section{B.3 $C_{N}=U S p(2 N)$}

The $N$ generators in the Cartan subalgebra of $U S p(2 N)$ are the following $2 N \times 2 N$ matrices,

$$
\mathbf{H}_{i}=\left(\begin{array}{cc}
\mathbf{B}_{\mathbf{i}} & \mathbf{0} \\
\mathbf{0} & -\mathbf{B}_{\mathbf{i}}^{\mathbf{t}}
\end{array}\right), \quad i=1,2, \ldots, N
$$

where

$$
\mathbf{B}_{\mathbf{i}}=\left(\begin{array}{ccccc}
w_{1}^{i} & & & & \\
& w_{2}^{i} & & & \\
& 0 & \ddots & 0 & \\
& & & w_{N-1}^{i} & \\
& & & & w_{N}^{i}
\end{array}\right), \quad i=1,2 \ldots N
$$


The weight vectors $\mathbf{w}_{k}(k=1,2, \ldots, N)$ form a complete set of orthogonal vectors in an $N$-dimensional Euclidean space and satisfy

$$
\mathbf{w}_{k} \cdot \mathbf{w}_{l}=0 ; \quad k \neq l ; \quad \mathbf{w}_{k} \cdot \mathbf{w}_{k}=\frac{1}{4(N+1)} .
$$

The root vectors of $U S p(2 N)$ group are $\alpha=\left\{ \pm 2 \mathbf{w}_{i}, \pm \mathbf{w}_{i} \pm \mathbf{w}_{j}\right\}$. The diagonal generators satisfy

$$
\operatorname{Tr} H_{i} H_{j}=\frac{1}{2(N+1)} \delta_{i j} .
$$

For the breaking $U S p(2 N) \rightarrow U S p(2(N-1)) \times U(1)$ the adjoint scalar VEV is

$$
\phi=\mathbf{h} \cdot \mathbf{H}, \quad \mathbf{h}=v \sqrt{4(N+1)}(0,0, \ldots, 1) .
$$

\section{B.4 $D_{N}=S O(2 N)$}

The $N$ generators in the Cartan subalgebra of the $S O(2 N)$ group can be chosen to be

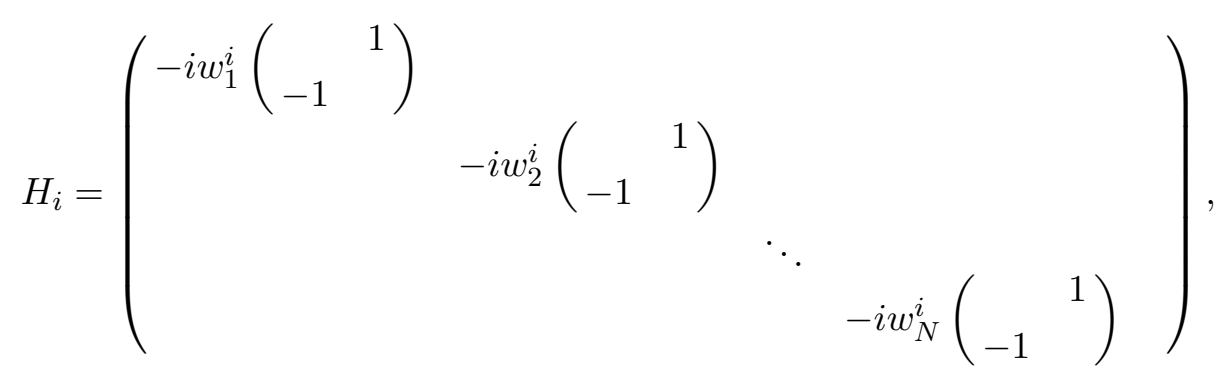

where $\mathbf{w}_{k}(k=1,2, \ldots, N)$ are the weight vectors of the fundamental representation, living in an $N$-dimensional Euclidean space and satisfying

$$
\mathbf{w}_{k} \cdot \mathbf{w}_{l}=0 ; \quad k \neq l ; \quad \mathbf{w}_{k} \cdot \mathbf{w}_{k}=\frac{1}{4(N-1)}:
$$

they form a complete set of orthogonal vectors. The root vectors of $S O(2 N)$ are $\alpha=\left\{ \pm \mathbf{w}_{i} \pm \mathbf{w}_{j}\right\}$. The diagonal generators satisfy

$$
\operatorname{Tr} H_{i} H_{j}=\frac{1}{2(N-1)} \delta_{i j}
$$

In the system with symmetry breaking $S O(2 N) \rightarrow S O(2 N-2) \times U(1)$ the adjoint scalar VEV takes the form

$$
\phi=\mathbf{h} \cdot \mathbf{H}, \quad \mathbf{h}=i v \sqrt{4(N-1)}(0,0, \ldots, 1) .
$$

